\title{
A METHOD TO CORRECT YIELD SURFACE DRIFT IN SOIL PLASTICITY UNDER MIXED CONTROL AND EXPLICIT INTEGRATION
}

\author{
HANS MATTSSON*, KENNET AXELSSON ${ }^{\dagger, \ddagger}$ AND MAREK KLISINSKI ${ }^{\dagger}$ \\ Department of Civil Engineering, Lulea University of Technology, S-97187 Luleå, Sweden
}

\begin{abstract}
SUMMARY
When applying an explicit integration algorithm in e.g. soil plasticity, the predicted stress point at the end of an elastoplastic increment of loading might not be situated on the updated current yield surface. This so-called yield surface drift could generally be held under control by using small integration steps. Another possibility, when circumstances might demand larger steps, is to adopt a drift correction method. In this paper, a drift correction method for mixed control in soil plasticity, under drained as well as undrained conditions, is proposed. By simulating triaxial tests in a Constitutive Driver, the capability and efficiency of this correction method, under different choices of implementation, have been analysed. It was concluded that the proposed drift correction method, for quite marginal additional computational cost, was able to correct successfully for yield surface drift giving results in close agreement to those obtained with a very large number of integration steps. (C) 1997 by John Wiley \& Sons, Ltd.
\end{abstract}

Int. j. numer. anal. methods geomech., vol. 21, 175-197 (1997)

(No. of Figures: $7 \quad$ No. of Tables: 2 No. of Refs: 8 )

Key words: soil plasticity; driff correction; mixed control; explicit integration

\section{INTRODUCTION}

Various methods for integration of constitutive relations in computational plasticity have been proposed in the literature. A brief review of such methods can be found in e.g. Reference 1. A basic classification of these methods into explicit and implicit categories can be done. Most of the implicit methods satisfy the incremental consistency condition, i.e. that the resulting stresses in plastic loading satisfy the yield criterion. This is generally not the case for explicit integration methods, based on a tangent formulation of the constitutive relations. When the yield surface, in such an explicit integration algorithm, is updated according to the hardening law at the end of an elastoplastic increment of loading the stress point corresponding to the predicted stress state does not have to be situated on the current yield surface. This so-called yield surface drift, can give rise to a cumulative discrepancy. The discrepancy can be decreased by the use of smaller loading

\footnotetext{
* Ph.D. Student

† Professor of Civil Engineering

${ }^{\ddagger}$ Correspondence to: K. Axelsson, Department of Civil Engineering, Luleå University of Technology, S-97187 Luleå, Sweden
} 
increments but not completely avoided. In this paper a method to correct for yield surface drift after each increment of loading, for explicit integration algorithms, is proposed.

Several methods to correct for yield surface drift have been described in the literature, see e.g. the paper by Potts and Gens ${ }^{2}$ in which five different drift correction methods are examined and compared. All these methods are formulated for a constitutive relationship in strain-driven format and describe different ways of correcting the stresses back to the yield surface. The strain-driven form of constitutive relations is predominant because it is the form used in displacement-based finite element codes. The drift correction methods discussed by Potts and Gens cannot, however, be applied to constitutive relations formulated in mixed control (control variables in stress and/or strain components) e.g. arising in soil plasticity for the calibration of model parameters at the constitutive level on the basis of conventional triaxial tests where the radial (confining) stress and the vertical strain are used as control variables. Again, other soil tests, e.g. the cubical cell test, corresponds to pure stress control. For simulating tests of these types, a drift correction method formulated for a general choice of control variables (stress, strain or mixed control) is needed when the corresponding constitutive equations are to be integrated explicitly. To the authors' knowledge, such a method has still not been proposed. In this paper, a drift correction method for constitutive relations in soil plasticity under mixed control and under drained and undrained behaviour will be formulated. The method is based on the presumptions that the control variables cannot be changed (only response variables and hardening parameters can be altered) and that any change in stress will cause an associated change in elastic strains, given by the elastic constitutive relation. It is the same approach as in the 'correct' projecting back method described by Potts and Gens. ${ }^{2}$ The proposed drift correction method has been implemented in a Constitutive Driver containing different soil models. The main purpose of this Constitutive Driver, which thus is based on mixed tangent relationship and the explicit Forward Euler integration algorithm, is to be used for calibration of model parameters at the constitutive level. For this approach the specimen in conventional and true triaxial apparatus is viewed as idealized in the meaning that the stress and strain state are assumed to be homogeneous.

It is assumed that the yield surface to be considered is represented in the effective stress space, even though other choices of state variables (stress, strain or mixed space) are possible. ${ }^{3}$ Further, in the formulation below, a distinction has been made between drained and undrained behaviours. In undrained behaviour the pore pressure is treated as a response variable while it takes a constant value in drained behaviour. The undrained condition is defined by one additional equation, the incompressibility constraint (frequently used for saturated soils) stating that no change in volume can occur under an undrained test.

The symbols and the formulation of the mixed tangent relationship under drained and undrained behaviour follows the paper by Runesson et al. ${ }^{4}$ That paper is highly recommended for additional information. The main application for such mixed tangent relations would be at the constitutive level together with an explicit integration algorithm; however it is possible that such relations could be incorporated in a finite element method in the future, e.g. for plane stress conditions, and then the presented drift correction method should be of interest even there. More research in that area would be of great value.

Matrix notation will be used throughout the paper. Stresses and strains are taken to be positive in compression. Further, a superimposed dot on the variables indicates a derivative with respect to a time-like evolution parameter (a rate), and could here be interpreted as an increment since plasticity is rate-independent. 


\section{BASIC EQUATIONS IN SOIL PLASTICITY}

In soil mechanics the effective stress principle expresses the relation between the effective stress $\boldsymbol{\sigma}$, the total stress $\mathbf{s}$ and the pore pressure $u$ as

$$
\dot{\boldsymbol{\sigma}}=\dot{\mathbf{s}}-\dot{u} \boldsymbol{\delta}
$$

where $\boldsymbol{\delta}$ is the Kronecker delta, here represented by a vector. It is assumed that all admissible stress states in the effective stress space are defined by the set $B$,

$$
B=\{\boldsymbol{\sigma} \mid F(\boldsymbol{\sigma}, \mathbf{\kappa}) \leqslant 0\}
$$

where the yield function $F(\boldsymbol{\sigma}, \mathbf{\kappa})$ in the usual way represents a limit between elastic states $F(\boldsymbol{\sigma}, \boldsymbol{\kappa})<0$ and plastic states $F(\boldsymbol{\sigma}, \boldsymbol{\kappa})=0$. The column vector $\boldsymbol{\kappa}$ contains hardening/softening parameters. The total strain increment vector $\dot{\boldsymbol{\varepsilon}}$ is divided into the two components,

$$
\dot{\varepsilon}=\dot{\varepsilon}^{\mathrm{e}}+\dot{\boldsymbol{\varepsilon}}^{\mathrm{p}}
$$

where $\dot{\boldsymbol{\varepsilon}}^{\mathrm{e}}$ is the elastic strain increment vector and $\dot{\boldsymbol{\varepsilon}}^{\mathrm{p}}$ is the plastic strain increment vector. The direction of the plastic strain increments is governed by the flow rule

$$
\dot{\boldsymbol{\varepsilon}}^{\mathrm{p}}=\dot{\lambda} \mathbf{m}
$$

where the vector $\mathbf{m}$ is commonly defined by the partial derivatives of a plastic potential function $G(\boldsymbol{\sigma}, \boldsymbol{\kappa})$ with respect to the stress components and $\dot{\lambda} \geqslant 0$ is a scalar multiplier. For a majority of elastoplastic models the evolution rule for $\boldsymbol{\kappa}$ can be expressed in the form

$$
\dot{\mathbf{\kappa}}=\mathbf{h}\left(\dot{\boldsymbol{\varepsilon}}^{\mathrm{p}}\right)=\dot{\lambda} \mathbf{h}(\mathbf{m})
$$

where the vector $\mathbf{h}$ contains state-dependent, first-order homogenous functions of the plastic strain increments. The consistency condition for a yield surface represented in the effective stress space becomes, with the notation $\mathbf{n}=\partial F / \partial \boldsymbol{\sigma}$,

$$
\dot{F}=\mathbf{n}^{\mathrm{T}} \dot{\boldsymbol{\sigma}}+\left(\frac{\partial F}{\partial \mathbf{\kappa}}\right)^{\mathrm{T}} \dot{\boldsymbol{\kappa}} \leqslant 0
$$

Utilizing equation (5) this condition takes the form

$$
\dot{F}=\mathbf{n}^{\mathrm{T}} \dot{\boldsymbol{\sigma}}-H \dot{\lambda} \leqslant 0
$$

where $H$ is the generalized plastic modulus under stress control,

$$
H=-\left(\frac{\partial F}{\partial \mathbf{\kappa}}\right)^{\mathrm{T}} \mathbf{h}(\mathbf{m})
$$

The plastic loading criteria, when $F=0$, can be summarized as

$$
\begin{array}{ll}
\dot{\lambda}>0, & \dot{F}=0, \text { plastic loading (P) } \\
\dot{\lambda}=0, & \dot{F}=0, \text { neutral loading (N) } \\
\dot{\lambda}=0, & \dot{F}<0, \text { elastic unloading (E) }
\end{array}
$$


or with (7)

$$
\begin{gathered}
\dot{\lambda}=\left(\frac{1}{H}\right) \mathbf{n}^{\mathrm{T}} \dot{\boldsymbol{\sigma}}>0(\mathrm{P}) \\
\mathbf{n}^{\mathrm{T}} \dot{\boldsymbol{\sigma}}=0(\mathrm{~N}) \\
\mathbf{n}^{\mathrm{T}} \dot{\boldsymbol{\sigma}}<0(\mathrm{E})
\end{gathered}
$$

It is important to observe that $H>0$ for an unambiguous loading criterion under the stress control.

The elastic tangential relationship between increments of stresses and increments of strains, is usually expressed either in stiffness form

$$
\dot{\boldsymbol{\sigma}}=\mathbf{D}^{\mathrm{e}} \dot{\boldsymbol{\varepsilon}}^{\mathrm{e}}
$$

or in compliance form

$$
\dot{\boldsymbol{\varepsilon}}^{\mathrm{e}}=\mathbf{C}^{\mathrm{e}} \dot{\boldsymbol{\sigma}}
$$

The appropriate elastic tangent relationship between the chosen control and response variables is essential for the present formulation. If $\boldsymbol{\sigma}$ and $\boldsymbol{\varepsilon}$ are decomposed into the energy conjugate portions $\sigma_{1}, \sigma_{2}$ and $\varepsilon_{1}, \varepsilon_{2}$, respectively, i.e.

$$
\boldsymbol{\sigma}=\left[\begin{array}{l}
\sigma_{1} \\
\sigma_{2}
\end{array}\right], \quad \varepsilon=\left[\begin{array}{l}
\varepsilon_{1} \\
\varepsilon_{2}
\end{array}\right]
$$

the elastic tangential relationship can be written in the form of the partitioned matrices

$$
\left[\begin{array}{c}
\dot{\boldsymbol{\sigma}}_{1} \\
\dot{\boldsymbol{\sigma}}_{2}
\end{array}\right]=\left[\begin{array}{cc}
\mathbf{D}_{11}^{\mathrm{e}} & \mathbf{D}_{12}^{\mathrm{e}} \\
\left(\mathbf{D}_{12}^{\mathrm{e}}\right)^{\mathrm{T}} & \mathbf{D}_{22}^{\mathrm{e}}
\end{array}\right]\left[\begin{array}{c}
\dot{\boldsymbol{\varepsilon}}_{1}^{\mathrm{e}} \\
\dot{\boldsymbol{\varepsilon}}_{2}^{\mathrm{e}}
\end{array}\right], \quad\left[\begin{array}{c}
\dot{\boldsymbol{\varepsilon}}_{1}^{\mathrm{e}} \\
\dot{\boldsymbol{\varepsilon}}_{2}^{\mathrm{e}}
\end{array}\right]=\left[\begin{array}{cc}
\mathbf{C}_{11}^{\mathrm{e}} & \mathbf{C}_{12}^{\mathrm{e}} \\
\left(\mathbf{C}_{12}^{\mathrm{e}}\right)^{\mathrm{T}} & \mathbf{C}_{22}^{\mathrm{e}}
\end{array}\right]\left[\begin{array}{c}
\dot{\boldsymbol{\sigma}}_{1} \\
\dot{\boldsymbol{\sigma}}_{2}
\end{array}\right]
$$

We can now, for instance, assume that $\boldsymbol{\sigma}_{1}$ and $\boldsymbol{\varepsilon}_{2}$ are control variables, whereas $\boldsymbol{\varepsilon}_{1}$ and $\boldsymbol{\sigma}_{2}$ are the associated response variables and partially invert equations (14) to obtain the mixed tangential elastic relationship

$$
\left[\begin{array}{c}
\dot{\boldsymbol{\varepsilon}}_{1}^{\mathrm{e}} \\
\dot{\boldsymbol{\sigma}}_{2}
\end{array}\right]=\left[\begin{array}{ll}
\mathbf{E}_{11}^{\mathrm{e}} & \mathbf{E}_{12}^{\mathrm{e}} \\
\mathbf{E}_{21}^{\mathrm{e}} & \mathbf{E}_{22}^{\mathrm{e}}
\end{array}\right]\left[\begin{array}{c}
\dot{\boldsymbol{\sigma}}_{1} \\
\dot{\boldsymbol{\varepsilon}}_{2}^{\mathrm{e}}
\end{array}\right]
$$

where

$$
\begin{aligned}
& \mathbf{E}_{11}^{\mathrm{e}}=\mathbf{C}_{11}^{\mathrm{e}}-\mathbf{C}_{12}^{\mathrm{e}}\left(\mathbf{C}_{22}^{\mathrm{e}}\right)^{-1}\left(\mathbf{C}_{12}^{\mathrm{e}}\right)^{\mathrm{T}}=\left(\mathbf{D}_{11}^{\mathrm{e}}\right)^{-1} \\
& \mathbf{E}_{12}^{\mathrm{e}}=\mathbf{C}_{12}^{\mathrm{e}}\left(\mathbf{C}_{22}^{\mathrm{e}}\right)^{-1}=-\left(\mathbf{D}_{11}^{\mathrm{e}}\right)^{-1} \mathbf{D}_{12}^{\mathrm{e}} \\
& \mathbf{E}_{21}^{\mathrm{e}}=-\left(\mathbf{E}_{12}^{\mathrm{e}}\right)^{\mathrm{T}} \\
& \mathbf{E}_{22}^{\mathrm{e}}=\left(\mathbf{C}_{22}^{\mathrm{e}}\right)^{-1}=\mathbf{D}_{22}^{\mathrm{e}}-\left(\mathbf{D}_{12}^{\mathrm{e}}\right)^{\mathrm{T}}\left(\mathbf{D}_{11}^{\mathrm{e}}\right)^{-1} \mathbf{D}_{12}^{\mathrm{e}}
\end{aligned}
$$

Relationships for other assumptions of control and response variables can easily be obtained by changing appropriate rows and columns in the tangential matrices.

The incompressibility constraint used for undrained behaviour is

$$
\dot{\boldsymbol{\varepsilon}}_{\mathrm{v}}=\boldsymbol{\delta}^{\mathrm{T}} \dot{\boldsymbol{\varepsilon}}=0
$$

where $\boldsymbol{\varepsilon}_{\mathrm{v}}$ is the volumetric strain. 


\section{TANGENT RELATIONSHIP UNDER MIXED CONTROL}

\subsection{Drained conditions}

Under drained conditions the pore pressure $u$ is constant and $\dot{\mathbf{s}}$ becomes equal to $\dot{\boldsymbol{\sigma}}$ which leads to the same formulation of the tangential relationship for either total stresses or effective stresses. When $\mathbf{s}_{1}=\sigma_{1}$ and $\varepsilon_{2}$ are chosen as the control variables the consistency condition (7) can be decomposed as

$$
\dot{F}=\mathbf{n}_{1}^{\mathrm{T}} \dot{\boldsymbol{\sigma}}_{1}+\mathbf{n}_{2}^{\mathrm{T}} \dot{\boldsymbol{\sigma}}_{2}-H \dot{\lambda} \leqslant 0
$$

In order to formulate this condition only in the control variables, it is necessary to express $\dot{\boldsymbol{\sigma}}_{2}$ in terms of these control variables via the mixed tangential elastic relationship (15). The consistency condition then becomes

$$
\dot{F}=\phi-K \dot{\lambda} \leqslant 0
$$

where $\phi$ is the loading function

$$
\phi=\hat{\mathbf{n}}_{1}^{\mathrm{T}} \dot{\boldsymbol{\sigma}}_{1}+\hat{\mathbf{n}}_{2}^{\mathrm{T}} \dot{\boldsymbol{\varepsilon}}_{2}
$$

and $K$ is the generalized plastic modulus under mixed control

$$
K=H+\mathbf{n}_{2}^{\mathrm{T}} \mathbf{E}_{22}^{\mathrm{e}} \mathbf{m}_{2}
$$

The 'transformed' gradients of $F$ are defined as

$$
\hat{\mathbf{n}}_{1}=\mathbf{n}_{1}-\mathbf{E}_{12}^{\mathrm{e}} \mathbf{n}_{2}, \quad \hat{\mathbf{n}}_{2}=\mathbf{E}_{22}^{\mathrm{e}} \mathbf{n}_{2}
$$

The loading criteria (9) now become

$$
\begin{gathered}
\dot{\lambda}=\left(\frac{1}{K}\right) \phi>0(\mathrm{P}) \\
\phi=0(\mathrm{~N}) \\
\phi<0(\mathrm{E})
\end{gathered}
$$

where $K>0$ for an unambiguous criterion. We have now enough background to solve for the plastic multiplier and formulate the mixed tangent relationship

$$
\left[\begin{array}{l}
\dot{\boldsymbol{\varepsilon}}_{1} \\
\dot{\boldsymbol{\sigma}}_{2}
\end{array}\right]=\left[\begin{array}{ll}
\mathbf{E}_{11} & \mathbf{E}_{12} \\
\mathbf{E}_{21} & \mathbf{E}_{22}
\end{array}\right]\left[\begin{array}{l}
\dot{\boldsymbol{\sigma}}_{1} \\
\dot{\boldsymbol{\varepsilon}}_{2}
\end{array}\right]
$$

If the 'transformed' flow vectors $\hat{\mathbf{m}}_{1}$ and $\hat{\mathbf{m}}_{2}$ are defined in a similar way as the 'transformed' gradients in equation (22), i.e.

$$
\hat{\mathbf{m}}_{1}=\mathbf{m}_{1}-\mathbf{E}_{12}^{\mathrm{e}} \mathbf{m}_{2}, \quad \hat{\mathbf{m}}_{2}=\mathbf{E}_{22}^{\mathrm{e}} \mathbf{m}_{2}
$$

the mixed tangent matrix under plastic loading can be written

$$
\left[\begin{array}{ll}
\mathbf{E}_{11} & \mathbf{E}_{12} \\
\mathbf{E}_{21} & \mathbf{E}_{22}
\end{array}\right]=\left[\begin{array}{ll}
\mathbf{E}_{11}^{\mathrm{e}} & \mathbf{E}_{12}^{\mathrm{e}} \\
\mathbf{E}_{21}^{\mathrm{e}} & \mathbf{E}_{22}^{\mathrm{e}}
\end{array}\right]+\frac{1}{K}\left[\begin{array}{cc}
\hat{\mathbf{m}}_{1} \hat{\mathbf{n}}_{1}^{\mathrm{T}} & \hat{\mathbf{m}}_{1} \hat{\mathbf{n}}_{2}^{\mathrm{T}} \\
-\hat{\mathbf{m}}_{2} \hat{\mathbf{n}}_{1}^{\mathrm{T}} & -\hat{\mathbf{m}}_{2} \hat{\mathbf{n}}_{2}^{\mathrm{T}}
\end{array}\right]
$$

which is reduced to

$$
\left[\begin{array}{ll}
\mathbf{E}_{11} & \mathbf{E}_{12} \\
\mathbf{E}_{21} & \mathbf{E}_{22}
\end{array}\right]=\left[\begin{array}{ll}
\mathbf{E}_{11}^{\mathrm{e}} & \mathbf{E}_{12}^{\mathrm{e}} \\
\mathbf{E}_{21}^{\mathrm{e}} & \mathbf{E}_{22}^{\mathrm{e}}
\end{array}\right]
$$

under elastic unloading. 


\subsection{Undrained conditions}

Again $\mathbf{s}_{1}$ and $\boldsymbol{\varepsilon}_{2}$ are chosen as control variables, which leaves $u, \boldsymbol{\varepsilon}_{1}$ and $\mathbf{s}_{2}$ as response variables. The incompressibility condition (17) is now decomposed into the form

$$
\dot{\boldsymbol{\varepsilon}}_{\mathrm{v}}=\boldsymbol{\delta}_{1}^{\mathrm{T}} \dot{\boldsymbol{\varepsilon}}_{1}+\boldsymbol{\delta}_{2}^{\mathrm{T}} \dot{\boldsymbol{\varepsilon}}_{2}=0
$$

By use of the effective stress principle and the flow rule,

$$
\left[\begin{array}{c}
\dot{\boldsymbol{\sigma}}_{1} \\
\dot{\boldsymbol{\sigma}}_{2}
\end{array}\right]=\left[\begin{array}{c}
\dot{\mathbf{s}}_{1} \\
\dot{\mathbf{s}}_{2}
\end{array}\right]-\dot{u}\left[\begin{array}{l}
\boldsymbol{\delta}_{1} \\
\boldsymbol{\delta}_{2}
\end{array}\right], \quad\left[\begin{array}{l}
\dot{\boldsymbol{\varepsilon}}_{1}^{\mathrm{p}} \\
\dot{\boldsymbol{\varepsilon}}_{2}^{\mathrm{p}}
\end{array}\right]=\dot{\lambda}\left[\begin{array}{l}
\mathbf{m}_{1} \\
\mathbf{m}_{2}
\end{array}\right]
$$

together with the elastic tangent relationship (15), the incompressibility condition (28) is rewritten as

$$
\dot{\boldsymbol{\varepsilon}}_{\mathrm{v}}=\overline{\boldsymbol{\delta}}_{1}^{\mathrm{T}} \dot{\mathbf{s}}_{1}+\overline{\boldsymbol{\delta}}_{2}^{\mathrm{T}} \dot{\boldsymbol{\varepsilon}}_{2}-c_{1}^{-1} \dot{u}+\dot{\lambda} \hat{m}_{1 \mathrm{v}}=0
$$

where the following convenient substitutions have been done

$$
\begin{gathered}
\overline{\boldsymbol{\delta}}_{1}=\mathbf{E}_{11}^{\mathrm{e}} \boldsymbol{\delta}_{1}, \quad \overline{\boldsymbol{\delta}}_{2}=\boldsymbol{\delta}_{2}-\mathbf{E}_{21}^{\mathrm{e}} \boldsymbol{\delta}_{1} \\
\hat{m}_{1 \mathrm{v}}=\boldsymbol{\delta}_{1}^{\mathrm{T}} \hat{\mathbf{m}}_{1} \\
c_{1}=1 / \boldsymbol{\delta}_{1}^{\mathrm{T}} \mathbf{E}_{11}^{\mathrm{e}} \boldsymbol{\delta}_{1}
\end{gathered}
$$

Here $\overline{\boldsymbol{\delta}}_{1}$ and $\overline{\boldsymbol{\delta}}_{2}$ are the adjusted Kronecker deltas and $\hat{m}_{1 \mathrm{v}}$ and $c_{1}$ are scalar variables. From eqn (30) we can solve for the pore pressure increment

$$
\dot{u}=c_{1}\left(\overline{\boldsymbol{\delta}}_{1}^{\mathrm{T}} \dot{\mathbf{s}}_{1}+\overline{\boldsymbol{\delta}}_{2}^{\mathrm{T}} \dot{\boldsymbol{\varepsilon}}_{2}+\dot{\lambda} \hat{m}_{1 \mathrm{v}}\right)
$$

Inserting eqn (34) into the effective stress principle (29) gives

$$
\dot{\boldsymbol{\sigma}}_{1}=\mathbf{D}_{11}^{\mathrm{e}} \mathbf{E}_{\mathrm{u} 11}^{\mathrm{e}} \dot{\mathbf{s}}_{1}-c_{1} \boldsymbol{\delta}_{1} \overline{\boldsymbol{\delta}}_{2}^{\mathrm{T}} \dot{\boldsymbol{\varepsilon}}_{2}-\dot{\lambda} c_{1} \hat{m}_{1 \mathrm{v}} \boldsymbol{\delta}_{1}
$$

where $\mathbf{E}_{\mathrm{u} 11}^{\mathbf{e}}$ is

$$
\mathbf{E}_{\mathrm{u} 11}^{\mathrm{e}}=\mathbf{E}_{11}^{\mathrm{e}}-c_{1} \overline{\boldsymbol{\delta}}_{1} \overline{\boldsymbol{\delta}}_{1}^{\mathrm{T}}
$$

In order to use the same formulation of the consistency condition as in equation (18) it is necessary to express $\dot{\boldsymbol{\sigma}}_{2}$ in terms of the control variables via the mixed elastic tangential relationship (15). After a few manipulations the expression

$$
\dot{F}=\mathbf{n}_{1}^{\mathrm{T}} \dot{\boldsymbol{\sigma}}_{1}+\mathbf{n}_{2}^{\mathrm{T}} \dot{\boldsymbol{\sigma}}_{2}-H \dot{\lambda}=\phi_{\mathbf{u}}-K_{\mathbf{u}} \dot{\lambda} \leqslant 0
$$

is obtained, where $\phi_{\mathrm{u}}$ is the undrained loading function

$$
\phi_{\mathrm{u}}=\hat{\mathbf{n}}_{1}^{\mathrm{T}} \mathbf{D}_{11}^{\mathrm{e}} \mathbf{E}_{\mathrm{u} 11}^{\mathrm{e}} \dot{\mathbf{s}}_{1}+\left(\hat{\mathbf{n}}_{2}-c_{1} \hat{n}_{1 \mathrm{v}} \overline{\boldsymbol{\delta}}_{2}\right)^{\mathrm{T}} \dot{\boldsymbol{\varepsilon}}_{2}=\hat{\mathbf{n}}_{\mathrm{u} 1}^{\mathrm{T}} \dot{\mathbf{s}}_{1}+\hat{\mathbf{n}}_{\mathrm{u} 2}^{\mathrm{T}} \dot{\boldsymbol{\varepsilon}}_{2}
$$

and $K_{\mathrm{u}}$ is the undrained generalized plastic modulus under mixed control

$$
K_{\mathrm{u}}=H+\mathbf{n}_{2}^{\mathrm{T}} \mathbf{E}_{22}^{\mathrm{e}} \mathbf{m}_{2}+c_{1} \hat{m}_{1 \mathrm{v}} \hat{n}_{1 \mathrm{v}}=K+c_{1} \hat{m}_{1 \mathrm{v}} \hat{n}_{1 \mathrm{v}}
$$

The introduced substitutions are

$$
\begin{gathered}
\hat{n}_{1 \mathrm{v}}=\boldsymbol{\delta}_{1}^{\mathrm{T}} \hat{\mathbf{n}}_{1} \\
\hat{\mathbf{n}}_{\mathrm{u} 1}=\hat{\mathbf{n}}_{1}-c_{1} \hat{n}_{1 \mathrm{v}} \overline{\boldsymbol{\delta}}_{1}, \quad \hat{\mathbf{n}}_{\mathrm{u} 2}=\hat{\mathbf{n}}_{2}-c_{1} \hat{n}_{1 \mathrm{v}} \overline{\boldsymbol{\delta}}_{2}
\end{gathered}
$$


where $\hat{n}_{1 \mathrm{v}}$ is a scalar and $\hat{\mathbf{n}}_{\mathrm{u} 1}$ and $\hat{\mathbf{n}}_{\mathrm{u} 2}$ are the undrained gradients. The loading criteria (9) now take the form

$$
\begin{gathered}
\dot{\lambda}=\left(\frac{1}{K_{\mathrm{u}}}\right) \phi_{\mathrm{u}}>0(\mathrm{P}) \\
\phi_{\mathrm{u}}=0(\mathrm{~N}) \\
\phi_{\mathrm{u}}<0(\mathrm{E})
\end{gathered}
$$

where $K_{\mathrm{u}}>0$ for an unambiguous criterion. We can now solve for the plastic multiplier and formulate the mixed tangent relationship

$$
\left[\begin{array}{l}
\dot{\boldsymbol{\varepsilon}}_{1} \\
\dot{\mathbf{s}}_{2}
\end{array}\right]=\left[\begin{array}{ll}
\mathbf{E}_{\mathrm{u} 11} & \mathbf{E}_{\mathrm{u} 12} \\
\mathbf{E}_{\mathrm{u} 21} & \mathbf{E}_{\mathrm{u} 22}
\end{array}\right]\left[\begin{array}{c}
\dot{\mathbf{s}}_{1} \\
\dot{\boldsymbol{\varepsilon}}_{2}
\end{array}\right]
$$

If the undrained plastic flow directions $\hat{\mathbf{m}}_{\mathrm{u} 1}$ and $\hat{\mathbf{m}}_{\mathrm{u} 2}$ are defined in a similar way as in equation (41), i.e.

$$
\hat{\mathbf{m}}_{\mathrm{u} 1}=\hat{\mathbf{m}}_{1}-c_{1} \hat{m}_{1 \mathbf{v}} \overline{\boldsymbol{\delta}}_{1}, \quad \hat{\mathbf{m}}_{\mathrm{u} 2}=\hat{\mathbf{m}}_{2}-c_{1} \hat{m}_{1 \mathbf{v}} \overline{\boldsymbol{\delta}}_{2}
$$

the mixed tangent matrix under plastic loading can be written

$$
\left[\begin{array}{ll}
\mathbf{E}_{\mathrm{u} 11} & \mathbf{E}_{\mathrm{u} 12} \\
\mathbf{E}_{\mathrm{u} 21} & \mathbf{E}_{\mathrm{u} 22}
\end{array}\right]=\left[\begin{array}{ll}
\mathbf{E}_{\mathrm{u} 11}^{\mathrm{e}} & \mathbf{E}_{\mathrm{u} 12}^{\mathrm{e}} \\
\mathbf{E}_{\mathrm{u} 21}^{\mathrm{e}} & \mathbf{E}_{\mathrm{u} 22}^{\mathrm{e}}
\end{array}\right]+\frac{1}{K_{\mathrm{u}}}\left[\begin{array}{cc}
\hat{\mathbf{m}}_{\mathrm{u} 1} \hat{\mathbf{n}}_{\mathrm{u} 1}^{\mathrm{T}} & \hat{\mathbf{m}}_{\mathrm{u} 1} \hat{\mathbf{n}}_{\mathrm{u} 2}^{\mathrm{T}} \\
-\hat{\mathbf{m}}_{\mathrm{u} 2} \hat{\mathbf{n}}_{\mathrm{u} 1}^{\mathrm{T}} & -\hat{\mathbf{m}}_{\mathrm{u} 2} \hat{\mathbf{n}}_{\mathrm{u} 2}^{\mathrm{T}}
\end{array}\right]
$$

In addition to $\mathbf{E}_{\mathbf{u} 11}^{\mathrm{e}}$ in equation (36) the undrained elastic moduli become

$$
\begin{gathered}
\mathbf{E}_{\mathrm{u} 12}^{\mathrm{e}}=\mathbf{E}_{12}^{\mathrm{e}}-c_{1} \overline{\boldsymbol{\delta}}_{1} \overline{\boldsymbol{\delta}}_{2}^{\mathrm{T}} \\
\mathbf{E}_{\mathrm{u} 21}^{\mathrm{e}}=-\left(\mathbf{E}_{\mathrm{u} 12}^{\mathrm{e}}\right)^{\mathrm{T}}=\mathbf{E}_{21}^{\mathrm{e}}+c_{1} \overline{\boldsymbol{\delta}}_{2} \overline{\boldsymbol{\delta}}_{1}^{\mathrm{T}} \\
\mathbf{E}_{\mathrm{u} 22}^{\mathrm{e}}=\mathbf{E}_{22}^{\mathrm{e}}+c_{1} \overline{\boldsymbol{\delta}}_{2} \overline{\boldsymbol{\delta}}_{2}^{\mathrm{T}}
\end{gathered}
$$

If $\dot{\lambda}$ is inserted into equation (34) the expression for the pore pressure increment $\dot{u}$ when $\phi_{\mathbf{u}}>0$ becomes

$$
\dot{u}=c_{1}\left(\overline{\boldsymbol{\delta}}_{1}+\frac{1}{K_{\mathrm{u}}} \hat{m}_{1 \mathrm{v}} \hat{\mathbf{n}}_{\mathrm{u} 1}\right)^{\mathrm{T}} \dot{\mathbf{s}}_{1}+c_{1}\left(\overline{\boldsymbol{\delta}}_{2}+\frac{1}{K_{\mathrm{u}}} \hat{m}_{1 \mathrm{v}} \hat{\mathbf{n}}_{\mathrm{u} 2}\right)^{\mathrm{T}} \dot{\boldsymbol{\varepsilon}}_{2}
$$

In the case of elastic loading, the mixed tangent matrix and the expression for $\dot{u}$ will be

$$
\begin{gathered}
{\left[\begin{array}{ll}
\mathbf{E}_{\mathrm{u} 11} & \mathbf{E}_{\mathrm{u} 12} \\
\mathbf{E}_{\mathrm{u} 21} & \mathbf{E}_{\mathrm{u} 22}
\end{array}\right]=\left[\begin{array}{ll}
\mathbf{E}_{\mathrm{u} 11}^{\mathrm{e}} & \mathbf{E}_{\mathrm{u} 12}^{\mathrm{e}} \\
\mathbf{E}_{\mathrm{u} 21}^{\mathrm{e}} & \mathbf{E}_{\mathrm{u} 22}^{\mathrm{e}}
\end{array}\right]} \\
\dot{u}=c_{1}\left(\overline{\boldsymbol{\delta}}_{1}^{\mathrm{T}} \dot{\mathbf{s}}_{1}+\overline{\boldsymbol{\delta}}_{2}^{\mathrm{T}} \dot{\boldsymbol{\varepsilon}}_{2}\right)
\end{gathered}
$$

\section{THE DRIFT CORRECTION METHOD}

In order to integrate numerically the derived constitutive tangential relationship under mixed control, equation (24) for drained behaviour or equation (43) for undrained behaviour, a constant tangential matrix has to be assumed for each small integration or iteration step. Considering explicit methods of integration, the evaluation of this tangential matrix might differ between different algorithms. These methods have in common that the stress point corresponding to the predicted stress state, at the end of an integration step in plastic loading, might not be situated on the yield surface updated according to the hardening law. In Figure 1 this yield surface drift is 


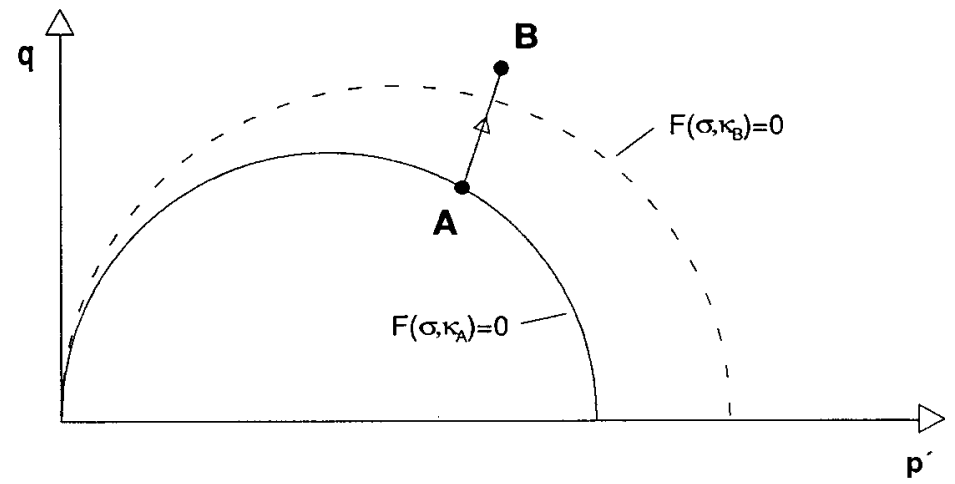

Figure 1. Sketch indicating yield surface drift

illustrated. The state of stress is here represented by the invariants $p^{\prime}=I_{1} / 3$ and $q=\left(3 J_{2}\right)^{1 / 2}$, where $I_{1}$ is the first invariant of the effective stress tensor and $J_{2}$ is the second invariant of the deviatoric stress tensor. Consider a step of plastic loading, at the start of which the stress point A is assumed to be situated on the yield surface $F\left(\boldsymbol{\sigma}, \boldsymbol{\kappa}_{\mathrm{A}}\right)=0$. At the end of the integration step, where the increments of the control variables are known and the increments of the response variables have been calculated, the stress and strain fields are updated. Further, the yield surface is updated to a new position $F\left(\boldsymbol{\sigma}, \boldsymbol{\kappa}_{\mathrm{B}}\right)=0$ according to the hardening law. Now, assume that the stress point $\mathrm{B}$, corresponding to the updated stress state, is not situated on the updated yield surface, i.e. $F\left(\boldsymbol{\sigma}_{\mathrm{B}}, \boldsymbol{\kappa}_{\mathrm{B}}\right) \neq 0$ and a so-called yield surface drift has taken place.

In an attempt to correct for such a yield surface drift, two basic presumptions are made:

(i) The control variables are not allowed to be changed, i.e. only the response variables and the hardening parameters can be adjusted.

(ii) Any change in effective stress will cause an associated change in the elastic strains, given by the elastic constitutive relation.

This implies that for the components of the total strain vector that are chosen as control variables, the correction process must be such that any change in the elastic strains is balanced by an equal and opposite change in the plastic strains, i.e.

$$
\begin{aligned}
& \Delta \varepsilon_{2}=\Delta \varepsilon_{2}^{\mathrm{e}}+\Delta \varepsilon_{2}^{\mathrm{p}}=0 \\
& \Delta \varepsilon_{2}^{\mathrm{e}}=-\Delta \varepsilon_{2}^{\mathrm{p}}
\end{aligned}
$$

When the corrections of the response variables and the hardening parameters have been determined, stresses, strains and hardening parameters in the state B are updated to a state C:

$$
\begin{gathered}
\mathbf{s}_{\mathrm{C}}=\mathbf{s}_{\mathrm{B}}+\Delta \mathbf{s} \\
u_{\mathrm{C}}=u_{\mathrm{B}}+\Delta u \\
\boldsymbol{\sigma}_{\mathrm{C}}=\boldsymbol{\sigma}_{\mathrm{B}}+\Delta \boldsymbol{\sigma} \\
\boldsymbol{\varepsilon}_{\mathrm{C}}=\boldsymbol{\varepsilon}_{\mathrm{B}}+\Delta \boldsymbol{\varepsilon} \\
\boldsymbol{\kappa}_{\mathrm{C}}=\boldsymbol{\kappa}_{\mathrm{B}}+\Delta \boldsymbol{\kappa}
\end{gathered}
$$

in which $F\left(\boldsymbol{\sigma}_{\mathrm{C}}, \boldsymbol{\kappa}_{\mathrm{C}}\right) \approx 0$. Corrections in equation (50) are denoted by the symbol $\Delta$. 


\subsection{Drained conditions}

Let the corrections of the effective stress and strain be denoted by $\Delta \sigma$ and $\Delta \varepsilon$, respectively. The mixed tangential elastic relationship, equation (15), can then be used to obtain

$$
\left[\begin{array}{c}
\Delta \boldsymbol{\varepsilon}_{1}^{\mathrm{e}} \\
\Delta \boldsymbol{\sigma}_{2}
\end{array}\right]=\left[\begin{array}{ll}
\mathbf{E}_{11}^{\mathrm{e}} & \mathbf{E}_{12}^{\mathrm{e}} \\
\mathbf{E}_{21}^{\mathrm{e}} & \mathbf{E}_{22}^{\mathrm{e}}
\end{array}\right]\left[\begin{array}{c}
\Delta \boldsymbol{\sigma}_{1} \\
\Delta \boldsymbol{\varepsilon}_{2}^{\mathrm{e}}
\end{array}\right]
$$

Because the control variables are not allowed to be changed, the constraints

$$
\Delta \sigma_{1}=0, \quad \Delta \varepsilon_{2}^{\mathrm{e}}=-\Delta \varepsilon_{2}^{\mathrm{p}}
$$

hold and equation (51) can be rewritten as

$$
\begin{aligned}
\Delta \boldsymbol{\varepsilon}_{1}^{\mathrm{e}} & =-\mathbf{E}_{12}^{\mathrm{e}} \Delta \boldsymbol{\varepsilon}_{2}^{\mathrm{p}} \\
\Delta \boldsymbol{\sigma}_{2} & =-\mathbf{E}_{22}^{\mathrm{e}} \Delta \boldsymbol{\varepsilon}_{2}^{\mathrm{p}}
\end{aligned}
$$

Further, it is assumed that the plastic strain corrections are proportional to the gradient of the plastic potential which gives

$$
\left[\begin{array}{c}
\Delta \boldsymbol{\varepsilon}_{1}^{\mathrm{p}} \\
\Delta \boldsymbol{\varepsilon}_{2}^{\mathrm{p}}
\end{array}\right]=\alpha\left[\begin{array}{l}
\mathbf{m}_{1} \\
\mathbf{m}_{2}
\end{array}\right]
$$

and an expression for the corrections of the response in equation (51) can be obtained as

$$
\begin{aligned}
\Delta \boldsymbol{\varepsilon}_{1}^{\mathrm{e}} & =-\alpha \mathbf{E}_{12}^{\mathrm{e}} \mathbf{m}_{2} \\
\Delta \boldsymbol{\sigma}_{2} & =-\alpha \mathbf{E}_{22}^{\mathrm{e}} \mathbf{m}_{2}=-\alpha \hat{\mathbf{m}}_{2}
\end{aligned}
$$

where the constant $\alpha$ is the only unknown and where $\hat{\mathbf{m}}$ contains the transformed gradients of the potential function, see equation (25). When the plastic strains are corrected, also the hardening parameters $\boldsymbol{\kappa}$ are corrected according to the evolution rule:

$$
\Delta \mathbf{\kappa}=\mathbf{h}\left(\Delta \boldsymbol{\varepsilon}^{\mathrm{p}}\right)=\alpha \mathbf{h}(\mathbf{m})
$$

The attempt here is to correct for yield surface drift and the corrected stress state should then satisfy the yield criterion

$$
F\left(\boldsymbol{\sigma}_{1 \mathrm{~B}}, \boldsymbol{\sigma}_{2 \mathrm{~B}}+\Delta \boldsymbol{\sigma}_{2}, \boldsymbol{\kappa}_{\mathrm{B}}+\Delta \boldsymbol{\kappa}\right)=0
$$

In order to do so, a Taylor series expansion neglecting terms in $\alpha^{2}$ and higher gives

$$
\alpha=\frac{F\left(\boldsymbol{\sigma}_{\mathrm{B}}, \boldsymbol{\kappa}_{\mathrm{B}}\right)}{\mathbf{n}_{2}^{\mathrm{T}} \hat{\mathbf{m}}_{2}-(\partial F / \partial \mathbf{\kappa})^{\mathrm{T}} \mathbf{h}(\mathbf{m})}
$$

or with (8) and (21)

$$
\alpha=\frac{F\left(\boldsymbol{\sigma}_{\mathrm{B}}, \boldsymbol{\kappa}_{\mathrm{B}}\right)}{K}
$$

where $K$ is thus the generalized plastic modulus under mixed control.

To calculate $\alpha$ (and later the corrections), the gradients $\partial F / \partial \boldsymbol{\sigma}, \partial G / \partial \boldsymbol{\sigma}, \partial F / \partial \boldsymbol{\kappa}$, the hardening functions $\mathbf{h}(\mathbf{m})$ and the elastic parameters in the elastic tangent matrix are required. Note that if a non-linear elastic response is assumed, the elastic tangent matrix becomes state dependent. Further, the functions $\mathbf{h}(\mathbf{m})$ could contain additional state parameters in addition to the vector m. The required quantities should, from a strictly mathematical point of view, be evaluated at 
the point B, but intuitively it feels like values at the beginning or end of the step or somewhere in between could be used if the integration increments are 'small' and the yield and potential functions are 'smooth enough', i.e. no rapid changes of the curvature of the corresponding surfaces occurs.

When $\alpha$ is calculated, the corrections of the response variables and of the hardening parameters are determined from equations (54)-(56):

$$
\left[\begin{array}{c}
\Delta \boldsymbol{\varepsilon}_{1} \\
\Delta \boldsymbol{\sigma}_{2}
\end{array}\right]=\alpha\left[\begin{array}{c}
\hat{\mathbf{m}}_{1} \\
-\hat{\mathbf{m}}_{2}
\end{array}\right], \quad \Delta \boldsymbol{\kappa}=\alpha \mathbf{h}(\mathbf{m})
$$

and a new, corrected, stress and strain field can be obtained according to equation (50).

Because higher-order terms have been neglected in the Taylor expansion, the corrected stresses and hardening parameters might not exactly satisfy the yield criterion $F(\boldsymbol{\sigma}, \mathbf{\kappa})=0$. If a single correction is not regarded to be sufficiently accurate, the correction procedure can be repeated until the stress state satisfies the yield criterion within a chosen tolerance (or the iteration stops after a maximum number of iterations, in case of slow or bad convergence). Such an iterative correction procedure could be formulated where the quantities $\partial F / \partial \boldsymbol{\sigma}, \partial G / \partial \boldsymbol{\sigma}, \partial F / \partial \mathbf{\kappa}, \mathbf{h}(\mathbf{m})$ and the elastic parameters are updated after each iteration or where the first choice of the quantities are used for all iterations. Obviously, there are many different ways to implement the drift correction method in a computer code. These possibilities will be examined closer below.

4.1.1. Special cases. The special cases of stress control and strain control, respectively, directly follows from the mixed control by the identifications $\Delta \sigma_{1}=\Delta \sigma\left(\Delta \varepsilon_{1}=\Delta \varepsilon\right)$ and $\Delta \varepsilon_{2}=\Delta \varepsilon\left(\Delta \sigma_{2}=\Delta \sigma\right)$.

In the case of stress control under drained conditions, the components in $\boldsymbol{\sigma}$ are treated as control variables and the correction constraint becomes $\Delta \boldsymbol{\sigma}=0$ and $\Delta \boldsymbol{\varepsilon}^{\mathrm{e}}=0$ follows from the basic presumptions. However, $\Delta \boldsymbol{\varepsilon}^{\mathrm{p}} \neq 0$ and the hardening parameters can be corrected in an attempt to satisfy the yield criterion $F(\boldsymbol{\sigma}, \boldsymbol{\kappa})=0$. Equation (58) will be reduced to

$$
\alpha=\frac{F\left(\boldsymbol{\sigma}_{\mathrm{B}}, \boldsymbol{\kappa}_{\mathrm{B}}\right)}{-(\partial F / \partial \mathbf{\kappa})^{\mathrm{T}} \mathbf{h}(\mathbf{m})}=\frac{F\left(\boldsymbol{\sigma}_{\mathrm{B}}, \boldsymbol{\kappa}_{\mathrm{B}}\right)}{H}
$$

where $H$ is the generalized plastic modulus under stress control. The corrections become

$$
\Delta \varepsilon=\alpha \mathbf{m}, \quad \Delta \mathbf{\kappa}=\alpha \mathbf{h}(\mathbf{m})
$$

In the case of strain control under drained conditions, the components in $\varepsilon$ are treated as control variables and the correction constraint becomes $\Delta \boldsymbol{\varepsilon}=0$ which implies that $\Delta \boldsymbol{\varepsilon}^{\mathrm{e}}=-\Delta \boldsymbol{\varepsilon}^{\mathrm{p}}$ from the basic presumptions. Equations (58) becomes

$$
\alpha=\frac{F\left(\boldsymbol{\sigma}_{\mathrm{B}}, \boldsymbol{\kappa}_{\mathrm{B}}\right)}{H+\mathbf{n}^{\mathrm{T}} \mathbf{D}^{\mathrm{e}} \mathbf{m}}
$$

and the corrections

$$
\Delta \boldsymbol{\sigma}=-\alpha \mathbf{D}^{\mathrm{e}} \mathbf{m}, \quad \Delta \boldsymbol{\kappa}=\alpha \mathbf{h}(\mathbf{m})
$$

Not surprisingly, this is the same result as Potts and Gens ${ }^{2}$ referred to as the 'correct' projecting back method. 


\subsection{Undrained conditions}

Under undrained conditions the part $\mathbf{s}_{1}$ of the total stress and $\boldsymbol{\varepsilon}_{2}$ of the total strain are chosen as control variables. Similar to the drained conditions, corrections are denoted by the symbol $\Delta$. The control variables are not allowed to be changed during the correction implying the constraints

$$
\Delta \mathbf{s}_{1}=0, \quad \Delta \varepsilon_{2}^{\mathrm{e}}=-\Delta \varepsilon_{2}^{\mathrm{p}}
$$

The corrections of the effective stresses, the total stresses and the pore pressure are related by the effective stress equation

$$
\left[\begin{array}{c}
\Delta \boldsymbol{\sigma}_{1} \\
\Delta \boldsymbol{\sigma}_{2}
\end{array}\right]=\left[\begin{array}{c}
\Delta \mathbf{s}_{1} \\
\Delta \mathbf{s}_{2}
\end{array}\right]-\Delta u\left[\begin{array}{l}
\boldsymbol{\delta}_{1} \\
\boldsymbol{\delta}_{2}
\end{array}\right]
$$

With equations (65) and (66), the mixed tangential elastic relationship, equation (51), can be rewritten as

$$
\begin{aligned}
\Delta \varepsilon_{1}^{\mathrm{e}} & =-\Delta u \overline{\boldsymbol{\delta}}_{1}-\mathbf{E}_{12}^{\mathrm{e}} \Delta \boldsymbol{\varepsilon}_{2}^{\mathrm{p}} \\
\Delta \boldsymbol{\sigma}_{2} & =-\Delta u \mathbf{E}_{21}^{\mathrm{e}} \boldsymbol{\delta}_{1}-\mathbf{E}_{22}^{\mathrm{e}} \Delta \boldsymbol{\varepsilon}_{2}^{\mathrm{p}}
\end{aligned}
$$

where $\overline{\boldsymbol{\delta}}$ is the adjusted Kronecker delta, see equation (31). The plastic strain corrections are assumed to be proportional to the gradient of the plastic potential

$$
\left[\begin{array}{c}
\Delta \boldsymbol{\varepsilon}_{1}^{\mathrm{p}} \\
\Delta \boldsymbol{\varepsilon}_{2}^{\mathrm{p}}
\end{array}\right]=\alpha\left[\begin{array}{l}
\mathbf{m}_{1} \\
\mathbf{m}_{2}
\end{array}\right]
$$

where $\alpha$ is treated as a constant. Equations (67) and (68) now give

$$
\begin{aligned}
\Delta \boldsymbol{\varepsilon}_{1}^{\mathrm{e}} & =-\Delta u \overline{\boldsymbol{\delta}}_{1}-\alpha \mathbf{E}_{12}^{\mathrm{e}} \mathbf{m}_{2} \\
\Delta \boldsymbol{\sigma}_{2} & =-\Delta u \mathbf{E}_{21}^{\mathrm{e}} \boldsymbol{\delta}_{1}-\alpha \hat{\mathbf{m}}_{2}
\end{aligned}
$$

where $\hat{\mathbf{m}}$ contains the 'transformed' gradients of the potential function, see equation (25). An expression for the pore pressure correction $\Delta u$ can be achieved from the incompressibility constraint $\Delta \varepsilon_{\mathrm{v}}=\boldsymbol{\delta}^{\mathrm{T}} \Delta \boldsymbol{\varepsilon}=0$ which, after a few manipulations, takes the form

$$
\Delta u=\alpha c_{1} \hat{m}_{1 \mathrm{v}}
$$

where $c_{1}$ is defined by equation (33) and $\hat{m}_{1 \mathrm{v}}$ by equation (32). Now the equations (66), (69) and (70) can be used to obtain an expression for the effective stress corrections

$$
\begin{aligned}
& \Delta \boldsymbol{\sigma}_{1}=-\alpha c_{1} \hat{m}_{1 \mathrm{v}} \boldsymbol{\delta}_{1} \\
& \Delta \boldsymbol{\sigma}_{2}=-\alpha\left(c_{1} \hat{m}_{1 \mathrm{v}} \boldsymbol{\delta}_{2}+\hat{\mathbf{m}}_{\mathrm{u} 2}\right)
\end{aligned}
$$

where the only unknown is the constant $\alpha$. Here $\hat{\mathbf{m}}_{\mathrm{u}}$ contains the undrained plastic flow directions, see equation (44). Due to the changes of the plastic strain, the hardening parameters $\boldsymbol{\kappa}$ are corrected as

$$
\Delta \mathbf{\kappa}=\alpha \mathbf{h}(\mathbf{m})
$$

The corrected effective stress state should satisfy the yield criterion, i.e.

$$
F\left(\boldsymbol{\sigma}_{1 \mathrm{~B}}+\Delta \boldsymbol{\sigma}_{1}, \boldsymbol{\sigma}_{2 \mathrm{~B}}+\Delta \boldsymbol{\sigma}_{2}, \boldsymbol{\kappa}_{\mathrm{B}}+\Delta \mathbf{\kappa}\right)=0
$$


A Taylor series expansion neglecting terms in $\alpha^{2}$ and higher gives

$$
\alpha=\frac{F\left(\boldsymbol{\sigma}_{\mathrm{B}}, \boldsymbol{\kappa}_{\mathrm{B}}\right)}{\mathbf{n}_{1}^{\mathrm{T}} c_{1} \hat{m}_{1 \mathrm{v}} \boldsymbol{\delta}_{1}+\mathbf{n}_{2}^{\mathrm{T}}\left(c_{1} \hat{m}_{1 \mathrm{v}} \boldsymbol{\delta}_{2}+\hat{\mathbf{m}}_{\mathrm{u} 2}\right)-(\partial F / \partial \mathbf{\kappa})^{\mathrm{T}} \mathbf{h}(\mathbf{m})}
$$

or with the undrained generalized plastic modulus under mixed control, $K_{\mathfrak{u}}$, according to equation (39)

$$
\alpha=\frac{F\left(\boldsymbol{\sigma}_{\mathrm{B}}, \boldsymbol{\kappa}_{\mathrm{B}}\right)}{K_{\mathrm{u}}}
$$

The discussion above concerning different ways of implementing the drift correction method in the case of drained conditions does also hold for undrained conditions. When $\alpha$ has been determined the corrections of the response variables and the hardening parameters are computed as

$$
\begin{aligned}
\Delta u & =\alpha c_{1} \hat{m}_{1 \mathrm{v}} \\
\Delta \boldsymbol{\sigma}_{1} & =-\Delta u \boldsymbol{\delta}_{1} \\
\Delta \boldsymbol{\sigma}_{2} & =-\Delta u \boldsymbol{\delta}_{2}-\alpha \hat{\mathbf{m}}_{\mathrm{u} 2} \\
\Delta \mathbf{s}_{2} & =-\alpha \hat{\mathbf{m}}_{\mathrm{u} 2} \\
\Delta \boldsymbol{\varepsilon}_{1} & =-\Delta u \overline{\boldsymbol{\delta}}_{1}+\alpha \hat{\mathbf{m}}_{1}=\alpha \hat{\mathbf{m}}_{\mathrm{u} 1} \\
\Delta \mathbf{K} & =\alpha \mathbf{h}(\mathbf{m})
\end{aligned}
$$

whereafter the stress and strain fields are updated according to equation (50).

4.2.1. Special cases. In the case of stress control under undrained conditions, the total stress components in $\mathbf{s}$ are treated as control variables and the correction constraint becomes $\Delta \mathbf{s}=0$. The special case of total stress control follows from the identification $\Delta \mathbf{s}_{1}=\Delta \mathbf{s}\left(\Delta \varepsilon_{1}=\Delta \varepsilon\right)$. Equation (74) becomes

$$
\alpha=\frac{F\left(\boldsymbol{\sigma}_{\mathrm{B}}, \boldsymbol{\kappa}_{\mathrm{B}}\right)}{H+c n_{\mathrm{v}} m_{\mathrm{v}}}
$$

where $H$ is the generalized plastic modulus under effective stress control and

$$
\begin{gathered}
c=1 / \boldsymbol{\delta}^{\mathrm{T}} \mathbf{C}^{\mathrm{e}} \boldsymbol{\delta} \\
n_{\mathrm{v}}=\boldsymbol{\delta}^{\mathrm{T}} \mathbf{n}, \quad m_{\mathrm{v}}=\boldsymbol{\delta}^{\mathrm{T}} \mathbf{m}
\end{gathered}
$$

The corrections will be

$$
\Delta u=\alpha c m_{\mathrm{v}}, \quad \Delta \boldsymbol{\sigma}=-\Delta u \boldsymbol{\delta}, \quad \Delta \boldsymbol{\varepsilon}=-\Delta u \mathbf{C}^{\mathrm{e}} \boldsymbol{\delta}+\alpha \mathbf{m}, \quad \Delta \mathbf{\kappa}=\alpha \mathbf{h}(\mathbf{m})
$$

Complete strain control is not possible for undrained behaviour. This is easy to realize because the incompressibility condition $\dot{\varepsilon}_{\mathrm{v}}=0$ will be overconstrained if not at least one normal strain component is left as a response variable.

\section{A STUDY OF SOME IMPLEMENTATION POSSIBILITIES}

In the Constitutive Driver used for this investigation, the drift correction method described above has been incorporated in the forward Euler explicit integration algorithm. The quantities $\partial F / \partial \boldsymbol{\sigma}$ 
$\partial G / \partial \boldsymbol{\sigma}, \partial F / \partial \mathbf{\kappa}, \mathbf{h}(\mathbf{m})$ and the elastic parameters needed in the drift correction method could be computed for the state either at point A or point B (see Figure 1) or for some intermediate point corresponding to a weighted average of the values at points A and B. However, it is not reasonable to analyse all these possibilities, so we will here concentrate on three different cases. The basic strategy for an integration step in plastic loading (when the Forward Euler integration algorithm is used together with the proposed drift correction method) for the different cases is as follows:

Case A: The tangential matrix is established at point A, the response variables are computed, the stress and strain fields are updated and the hardening parameters are updated according to the hardening law. Thereafter, the generalized plastic modulus, $K$ or $K_{u}$, and the quantities $\partial F / \partial \boldsymbol{\sigma}$, $\partial G / \partial \boldsymbol{\sigma}$, and the elastic parameters already computed at point $\mathrm{A}$ are used in the drift correction method and the stress and strain fields and the hardening parameters are corrected for drift. The additional state dependence in the function $\mathbf{h}(\mathbf{m})$ is however computed at point B for the corrections of the hardening parameters.

Case B: The quantities $\partial F / \partial \boldsymbol{\sigma}, \partial G / \partial \boldsymbol{\sigma}, \partial F / \partial \mathbf{\kappa}, \mathbf{h}(\mathbf{m})$ and the elastic tangent matrix are computed at point B. From that the generalized plastic modulus, $K$ or $K_{\mathrm{u}}$, is calculated. Now these values are used in the drift correction method to correct the stress and strain fields and the hardening parameters. The values of $\partial F / \partial \boldsymbol{\sigma}, \partial G / \partial \boldsymbol{\sigma}, \partial F / \partial \mathbf{\kappa}, K$ or $K_{\mathbf{u}}$ and the elastic tangent matrix previously computed at point $\mathrm{B}$ are now used to establish the tangential matrix. The response variables are computed and the stress field, strain field and hardening parameters are updated.

Case AB: The tangential matrix is established at point A, the response variables are computed, the stress and strain fields are updated and the hardening parameters are updated according to the hardening law. After that all the parameters needed in the drift correction method are computed at point $\mathrm{B}$ and corrections are performed.

It can be seen that the main difference between the cases A and B is only a matter when the corrections are peformed. In case $\mathrm{A}$ the corrections are made at the end of the current integration step and in case B at the beginning of the following step. The computer time needed for the computations will be equal for the cases $\mathrm{A}$ and $\mathrm{B}$ but for the case $\mathrm{AB}$ additional time is needed to compute the quantities $\partial F / \partial \boldsymbol{\sigma}, \partial G / \partial \boldsymbol{\sigma}, \partial F / \partial \mathbf{\kappa}, \mathbf{h}(\mathbf{m})$ and the elastic tangential matrix at point $\mathrm{B}$ before performing the corrections.

In order to investigate the impact of drift correction in the three different cases discussed, results for computations with comparatively few integration steps when adopting drift correction is compared with an 'exact' solution utilizing a very large number of integration steps. Computations both with single correction and with repeated corrections are examined.

To be able to compare the results from these computations, a norm is chosen based on the average value of the closest discrete Euclidean distances, in a seven-dimensional space spanned by the principal total stresses, the principal strains and the pore pressure, between state values at each of the points corresponding to the computation with drift correction and the values corresponding to an 'exact' solution. The Euclidean distance between two such points is

$$
d=\left[\frac{1}{s_{0}^{2}} \sum_{i=1}^{3}\left(s_{i}^{\mathrm{dcm}}-s_{i}^{\text {exact }}\right)^{2}+\frac{1}{\varepsilon_{0}^{2}} \sum_{i=1}^{3}\left(\varepsilon_{i}^{\mathrm{dcm}}-\varepsilon_{i}^{\text {exact }}\right)^{2}+\frac{1}{u_{0}^{2}}\left(u^{\mathrm{dcm}}-u^{\text {exact }}\right)^{2}\right]^{1 / 2}
$$


where $s_{0}, \varepsilon_{0}$ and $u_{0}$ are scaling factors necessary for the comparison of stresses and strains. The scaling factors are chosen as the maximum absolute value of all the total stresses, strains and pore pressures, respectively, in the discrete points involved in the computations

$$
\begin{aligned}
s_{0} & =\max \left(\left|s_{1}\right|,\left|s_{2}\right|,\left|s_{3}\right|\right) \\
\varepsilon_{0} & =\max \left(\left|\varepsilon_{1}\right|,\left|\varepsilon_{2}\right|,\left|\varepsilon_{3}\right|\right) \\
u_{0} & =\max |u|
\end{aligned}
$$

giving the inequalities

$$
\begin{aligned}
& -1 \leqslant \frac{s_{1}}{s_{0}}, \frac{s_{2}}{s_{0}}, \frac{s_{3}}{s_{0}} \leqslant 1 \\
& -1 \leqslant \frac{\varepsilon_{1}}{\varepsilon_{0}}, \frac{\varepsilon_{2}}{\varepsilon_{0}}, \frac{\varepsilon_{3}}{\varepsilon_{0}} \leqslant 1 \\
& -1 \leqslant \frac{u}{u_{0}} \leqslant 1
\end{aligned}
$$

Since only discrete points are known in reality for both the computations to be compared, the distance is measured between such points and the closest distance is computed as the height of a triangle. The final norm will be

$$
E=\frac{1}{k+1}\left[\sum_{j=1}^{k} d_{j}+d_{\mathrm{t}}\right]
$$

where $k$ is the number of points in the computation with the drift correction method and $d_{\mathrm{t}}$ is the Euclidean distance between the termination points, which do achieve extra attention in this way. More information about norms of this kind can be found in, for example, Reference 5 or Reference 6.

In the following, the proposed drift correction method is demonstrated for the above discussed cases A, B and AB. By using the Constitutive Driver and, for simplicity, applying the modified Cam Clay model, see Reference 7, ten loading programs have been investigated, see Table I. The state parameters $p_{\mathrm{c}}^{\prime}$ and $v$ (in Table I) stands for the isotropic effective mean stress (that is the hardening parameter) and the specific volume, respectively. The tests 1-6 (in Table I) are numerical simulations of conventional triaxial compression tests while the tests $7-10$ are numerical simulations of tests that only can be performed in a true triaxial apparatus. In the tests, a linear variation of the control parameters between the initial and final values is assumed.

Each of the ten loading programs was performed with single correction and with repeated corrections for the implementation cases $\mathrm{A}, \mathrm{B}$ and $\mathrm{AB}$. Computations without using drift correction were also performed for the loading programs. For all the alternatives the norm in equation (83) was computed, see Table II. The 'exact' solution that is included in the norm was carried out with 100,000 integration steps without drift correction. All loading programs were performed with 30 as well as 100 integration steps. To compensate for the additional computation time needed in single correction for the case $\mathrm{AB}$ compared to the cases $\mathrm{A}$ and $\mathrm{B}$, computations with 26 and 85 integration steps were also performed for the case $\mathrm{AB}$, with the motivation that the computation time for this case then will be approximately the same as for cases A and $\mathrm{B}\left(\right.$ Time $\left.^{\mathrm{AB}} \approx \mathrm{Time}^{\mathrm{A} \text { or } \mathrm{B}} / 0.85\right)$. The repeated corrections were carried out where the quantities 
Table I. Loading programs with the modified Cam Clay model. (Model parameters chosen: $\kappa=0 \cdot 05$, $v^{\prime}=0 \cdot 3, \lambda=0 \cdot 2, M=1 \cdot 0, N=2 \cdot 9$. For definition of symbols used, see e.g. Reference 8 .)

\begin{tabular}{|c|c|c|c|c|c|c|c|c|c|c|c|c|c|}
\hline \multirow[t]{2}{*}{ Test } & \multirow{2}{*}{$\begin{array}{c}\text { Drained } \\
\text { [0] or } \\
\text { undrained } \\
{[1]}\end{array}$} & \multicolumn{6}{|c|}{ Initial Conditions } & \multicolumn{6}{|c|}{ End conditions of control parameters } \\
\hline & & $\begin{array}{c}p_{c}^{\prime} \\
(\mathrm{kPa})\end{array}$ & $\begin{array}{c}S_{1} \\
(\mathrm{kPa})\end{array}$ & $\begin{array}{c}S_{2} \\
(\mathrm{kPa})\end{array}$ & $\begin{array}{c}S_{3} \\
(\mathrm{kPa})\end{array}$ & $\begin{array}{c}u \\
(\mathrm{kPa})\end{array}$ & $v$ & $\begin{array}{c}s_{1} \\
(\mathrm{kPa})\end{array}$ & $\begin{array}{c}s_{2} \\
(\mathrm{kPa})\end{array}$ & $\begin{array}{c}S_{3} \\
(\mathrm{kPa})\end{array}$ & $\begin{array}{c}\varepsilon_{1} \\
(\%)\end{array}$ & $\begin{array}{c}\varepsilon_{2} \\
(\%)\end{array}$ & $\begin{array}{c}\varepsilon_{3} \\
(\%)\end{array}$ \\
\hline 1 & 0 & 100 & 100 & 100 & 100 & 0 & 1.98 & - & 100 & 100 & 30 & - & - \\
\hline 2 & 1 & 100 & 100 & 100 & 100 & 0 & 1.98 & - & 100 & 100 & 10 & - & - \\
\hline 3 & 0 & 125 & 100 & 100 & 100 & 0 & $1 \cdot 945$ & - & 100 & 100 & 30 & - & - \\
\hline 4 & 1 & 125 & 100 & 100 & 100 & 0 & $1 \cdot 945$ & - & 100 & 100 & 10 & - & - \\
\hline 5 & 0 & 500 & 100 & 100 & 100 & 0 & $1 \cdot 738$ & - & 100 & 100 & 30 & - & - \\
\hline 6 & 1 & 500 & 100 & 100 & 100 & 0 & $1 \cdot 738$ & - & 100 & 100 & 10 & - & - \\
\hline 7 & 0 & 100 & 100 & 100 & 100 & 0 & 1.98 & - & - & - & 8 & -4 & 4 \\
\hline 8 & 0 & 100 & 100 & 100 & 100 & 0 & 1.98 & 180 & 140 & 125 & - & - & - \\
\hline 9 & 1 & 100 & 100 & 100 & 100 & 0 & 1.98 & 125 & 75 & - & - & - & 0 \\
\hline 10 & 1 & 100 & 100 & 100 & 100 & 0 & 1.98 & 160 & 130 & 120 & - & - & - \\
\hline
\end{tabular}

Table II. Norm values in \%

\begin{tabular}{|c|c|c|c|c|c|c|c|c|c|}
\hline \multirow[t]{3}{*}{ Test } & \multirow[t]{3}{*}{ Int. steps } & \multirow{3}{*}{$\begin{array}{l}\text { Without } \\
\text { DCM }\end{array}$} & \multicolumn{4}{|c|}{ Single correction } & \multirow{2}{*}{\multicolumn{3}{|c|}{$\begin{array}{l}\text { Repeated corrections until } \\
\qquad F(\boldsymbol{\sigma}, \boldsymbol{\kappa}) \leqslant 1 \cdot 0 \mathrm{E}-10\end{array}$}} \\
\hline & & & \multirow[t]{2}{*}{ Case A } & \multirow[t]{2}{*}{ Case B } & \multirow[t]{2}{*}{ Case AB } & \multirow{2}{*}{$\begin{array}{l}\text { Case AB } \\
\text { int. steps } \\
\quad \times 0.85\end{array}$} & & & \\
\hline & & & & & & & Case A & Case B & Case AB \\
\hline \multirow[t]{2}{*}{1} & 30 & $2 \cdot 7051$ & $1 \cdot 5353$ & 0.9299 & 0.9836 & $1 \cdot 1217$ & $1 \cdot 3069$ & $0 \cdot 9385$ & 0.9956 \\
\hline & 100 & $0 \cdot 6548$ & $0 \cdot 3382$ & $0 \cdot 3056$ & $0 \cdot 3129$ & $0 \cdot 3668$ & $0 \cdot 3329$ & $0 \cdot 3057$ & $0 \cdot 3130$ \\
\hline \multirow[t]{2}{*}{2} & 30 & $5 \cdot 5007$ & $1 \cdot 4027$ & $1 \cdot 3803$ & $1 \cdot 1600$ & $1 \cdot 3106$ & $1 \cdot 4020$ & $1 \cdot 3807$ & $1 \cdot 1618$ \\
\hline & 100 & $1 \cdot 5420$ & $0 \cdot 4029$ & $0 \cdot 3982$ & $0 \cdot 3820$ & $0 \cdot 4465$ & $0 \cdot 4028$ & $0 \cdot 3982$ & $0 \cdot 3820$ \\
\hline \multirow[t]{2}{*}{3} & 30 & $1 \cdot 0047$ & 0.5833 & $0 \cdot 5831$ & 0.5763 & $0 \cdot 6612$ & $0 \cdot 5823$ & 0.5831 & 0.5764 \\
\hline & 100 & $0 \cdot 2889$ & $0 \cdot 1692$ & $0 \cdot 1686$ & $0 \cdot 1686$ & $0 \cdot 1996$ & $0 \cdot 1692$ & $0 \cdot 1686$ & $0 \cdot 1686$ \\
\hline \multirow[t]{2}{*}{4} & 30 & $1 \cdot 6944$ & $1 \cdot 0418$ & $1 \cdot 0283$ & $1 \cdot 0150$ & $1 \cdot 1480$ & $1 \cdot 0414$ & $1 \cdot 0284$ & $1 \cdot 0151$ \\
\hline & 100 & $0 \cdot 4907$ & $0 \cdot 3098$ & $0 \cdot 3086$ & $0 \cdot 3076$ & $0 \cdot 3641$ & $0 \cdot 3098$ & $0 \cdot 3086$ & $0 \cdot 3076$ \\
\hline \multirow[t]{2}{*}{5} & 30 & $0 \cdot 3799$ & $0 \cdot 4406$ & 0.4390 & $0 \cdot 4406$ & $0 \cdot 4094$ & $0 \cdot 4407$ & 0.4390 & $0 \cdot 4406$ \\
\hline & 100 & 0.0907 & $0 \cdot 1057$ & $0 \cdot 1056$ & $0 \cdot 1056$ & $0 \cdot 1246$ & $0 \cdot 1057$ & $0 \cdot 1056$ & $0 \cdot 1056$ \\
\hline \multirow[t]{2}{*}{6} & 30 & $0 \cdot 3692$ & $0 \cdot 8487$ & $0 \cdot 7862$ & $0 \cdot 8291$ & $0 \cdot 8660$ & $0 \cdot 8501$ & $0 \cdot 7862$ & $0 \cdot 8291$ \\
\hline & 100 & 0.0738 & $0 \cdot 2002$ & $0 \cdot 1942$ & $0 \cdot 1984$ & $0 \cdot 2207$ & $0 \cdot 2002$ & $0 \cdot 1942$ & $0 \cdot 1984$ \\
\hline \multirow[t]{2}{*}{7} & 30 & $0 \cdot 6171$ & 0.7576 & 0.7428 & 0.7339 & $0 \cdot 8494$ & $0 \cdot 7563$ & 0.7429 & 0.7342 \\
\hline & 100 & $0 \cdot 1670$ & $0 \cdot 2185$ & $0 \cdot 2162$ & $0 \cdot 2167$ & $0 \cdot 2552$ & $0 \cdot 2185$ & $0 \cdot 2162$ & $0 \cdot 2167$ \\
\hline \multirow[t]{2}{*}{8} & 30 & $0 \cdot 6037$ & 0.6577 & 0.6465 & 0.6453 & $0 \cdot 7498$ & $0 \cdot 6574$ & 0.6465 & 0.6453 \\
\hline & 100 & $0 \cdot 1769$ & $0 \cdot 1948$ & $0 \cdot 1936$ & $0 \cdot 1935$ & $0 \cdot 2190$ & $0 \cdot 1948$ & $0 \cdot 1936$ & $0 \cdot 1935$ \\
\hline \multirow[t]{2}{*}{9} & 30 & $1 \cdot 0225$ & 0.4322 & $0 \cdot 3965$ & $0 \cdot 4051$ & $0 \cdot 4729$ & 0.4329 & 0.3965 & $0 \cdot 4051$ \\
\hline & 100 & $0 \cdot 2672$ & $0 \cdot 1221$ & $0 \cdot 1143$ & $0 \cdot 1198$ & $0 \cdot 1399$ & $0 \cdot 1221$ & $0 \cdot 1143$ & $0 \cdot 1198$ \\
\hline \multirow[t]{2}{*}{10} & 30 & $0 \cdot 3027$ & $0 \cdot 2258$ & $0 \cdot 2197$ & $0 \cdot 2111$ & $0 \cdot 2465$ & $0 \cdot 2258$ & $0 \cdot 2197$ & $0 \cdot 2111$ \\
\hline & 100 & $0 \cdot 0748$ & 0.0619 & 0.0608 & 0.0606 & $0 \cdot 0713$ & 0.0619 & 0.0608 & 0.0606 \\
\hline
\end{tabular}


described in the cases $\mathrm{A}, \mathrm{B}$ and $\mathrm{AB}$, respectively, were fixed for the entire iteration. The corrections proceeded until $F(\boldsymbol{\sigma}, \boldsymbol{\kappa}) \leqslant 1.0 \times 10^{-10}$.

The total error obtained in an explicit integration algorithm, here forward Euler, could be decomposed into an error caused by the use of gradually constant tangent matrices and an error caused by the yield surface drift which primarily arises from the use of the first-order consistency condition. These errors, however, are linked together since the consistency condition is used to establish the tangential matrix. Further, both types of error will decrease with an increasing number of integration steps. The drift correction method is able to reduce (or eliminate) only the errors arising from the yield surface drift. This correction will, in turn, have an influence on the subsequent tangential matrices and thus indirectly on the other type of error. This leads to the conclusion that we cannot generally expect that a computation in which drift correction is used is closer to the 'exact' solution than a computation without use of drift correction. This is noticed in tests 5-8 where the norm values are slightly smaller for computations where no drift correction is used in comparison to computations utilizing drift correction. However, these small differences might be of little practical importance. For tests 1-4 and tests 9-10 the norm values for computations with the drift correction method are smaller compared to calculations without use of drift correction. Especially for the tests 1 and 2, where much drift is produced when the drift correction method is not applied, a considerable improvement is achieved when applying the drift correction method.

If the implementation types $\mathrm{A}, \mathrm{B}$ and $\mathrm{AB}$ of the drift correction method are compared (for the same computer effort in single correction), it is seen that these give rise to quite small differences in predictions. This is true for all tests except for the test 1 (with 30 integration steps) where a somewhat larger norm value for the case A is obtained. However, the implementation case $\mathrm{B}$ yields the smallest norm values for all tests, except for the tests 2 and 5 (with 30 integration steps). The reason why the case $\mathrm{B}$ gives somewhat better results than the cases $\mathrm{A}$ and $\mathrm{AB}$ (when the same computer effort is compared) is most likely that the values of $\partial F / \partial \boldsymbol{\sigma}, \partial G / \partial \boldsymbol{\sigma}, \partial F / \partial \mathbf{\kappa}, K$ or $K_{\mathrm{u}}$ and the elastic tangent matrix in this case are established in a state somewhere inside the integration step which will lead to a faster convergence to the exact solution. If that is a correct assumption, the case B can only be expected to give better results for radial loading mainly, corresponding to the loading programs in Table I. The small difference between the norm values when repeated corrections are compared to single correction indicates that the additional computer effort needed in the procedure of repeated corrections will be of more economical use in more integration steps together with single correction, at least for the type of loading programs in Table I. It should be pointed out that Potts and Gens ${ }^{2}$ performed analyses of drift correction only in strain control (see equations (63) and (64)) where the quantities $\partial F / \partial \boldsymbol{\sigma}, \partial G / \partial \boldsymbol{\sigma}$ and $\partial F / \partial \mathbf{K}$ were evaluated at both the beginning and the end of the step in a repeated correction procedure, for a boundary value problem using a form of the modified Cam Clay model, and came to the conclusion that it makes little difference to the predictions where the quantities were evaluated.

\section{NUMERICAL EXAMPLES}

To get a better picture of how the proposed drift correction method behaves, tests 1 and 2 in Table I will now be examined in more detail. In addition, a third non-conventional test will be simulated by the Constitutive Driver using the same soil model and model parameters as in 
Table I. In all examples the implementation case B with single correction has been used for the drift correction method. The results are shown in plots with the invariants

$$
\begin{aligned}
p^{\prime} & =\left(\sigma_{1}+\sigma_{2}+\sigma_{3}\right) / 3 \\
q & =\left(\frac{\left(\sigma_{1}-\sigma_{2}\right)^{2}+\left(\sigma_{2}-\sigma_{3}\right)^{2}+\left(\sigma_{1}-\sigma_{3}\right)^{2}}{2}\right)^{1 / 2} \\
\varepsilon_{p} & =\varepsilon_{1}+\varepsilon_{2}+\varepsilon_{3} \\
\varepsilon_{q} & =\frac{2^{1 / 2}}{3}\left(\left(\varepsilon_{1}-\varepsilon_{2}\right)^{2}+\left(\varepsilon_{2}-\varepsilon_{3}\right)^{2}+\left(\varepsilon_{1}-\varepsilon_{3}\right)^{2}\right)^{1 / 2}
\end{aligned}
$$

where $\sigma_{1}, \sigma_{2}$ and $\sigma_{3}$ are the principal effective stresses and $\varepsilon_{1}, \varepsilon_{2}$ and $\varepsilon_{3}$ are the principal strains. For the simulations with only few integration steps, plots of the absolute value of the modified Cam Clay yield function

$$
F(\boldsymbol{\sigma}, \mathbf{\kappa})=q^{2}+M^{2}\left(p^{\prime 2}-p^{\prime} p_{\mathrm{c}}^{\prime}\right)
$$

against the number of integration steps are also shown. The value of $|F(\boldsymbol{\sigma}, \boldsymbol{\kappa})|$ is saved after the correction, in case $\mathrm{B}$, when the drift correction method is used and in the corresponding place in the algorithm when no drift correction is used.

\subsection{Example 1}

In this example the test 1 in Table $\mathrm{I}$, a simulation of a conventional drained triaxial compression test on a normally consolidated clay, is examined in further detail. In Figure 2 the responses in $q: \varepsilon_{q^{-}}, \varepsilon_{p}: \varepsilon_{q^{-}}, q: p^{\prime}$ - and $\varepsilon_{p}: p^{\prime}$-planes are shown for an 'exact' computation (with 100,000 integration increments) and for computations with 30 integration increments with and without use of the drift correction method. The plot of $|F(\boldsymbol{\sigma}, \mathbf{\kappa})|$ against the number of integration steps is presented in Figure 3. It is seen that serious drift from the yield surface occurs when the drift correction method is not used. However, when using this method, the final corrected solution is seen to be very close to the 'exact' solution. Without drift correction the computation leads to e.g. an overestimated shear strength (see Figure 2). From Figure 3 it is seen that most of the drift takes place during the first integration step whereafter the rate of produced drift gradually decreases. The reason for this is that the gradient $\partial F / \partial q$ initially is equal to zero (when $q=0$ ) for normally consolidated Cam Clay, but as soon as the loading proceeds this gradient obtains a non-zero value since the stress path in a conventional drained triaxial compression test is governed by the relation $\Delta q / \Delta p^{\prime}=3$.

\subsection{Example 2}

In this example the test 2 in Table I is further examined. Figures 4 and 5 show some results from this simulation of a conventional undrained triaxial compression test on a normally consolidated clay. Similar to the previous example, serious drift is obtained when the drift correction method is not used. However, this method is able to improve the solution so that it almost corresponds to the 'exact' solution. It is seen that the stress path in the $q: p^{\prime}$-plane in the beginning of the 

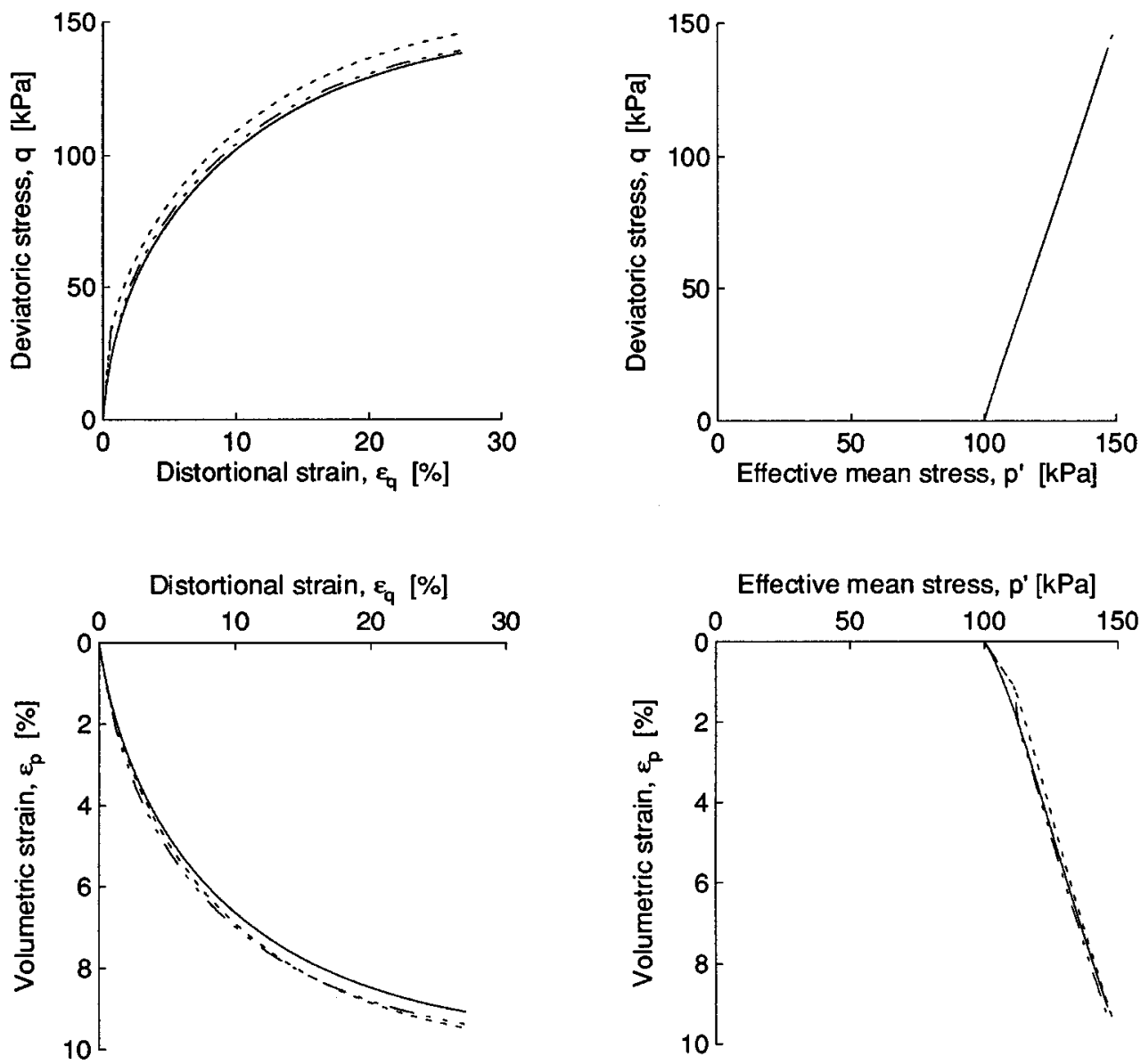

\section{— Forward Euler with 100000 integration increments \\ Forward Euler with 30 integration increments \\ -..- Forward Euler with 30 integration increments + drift correction method}

Figure 2. A simulation of a conventional drained triaxial compression test on a normally consolidated clay with the modified Cam Clay model

deviatoric loading moves vertically upwards at constant $p^{\prime}$. This is due to the fact that the first integration step in the forward Euler algorithm is treated as neutral loading $\left(\phi_{\mathbf{u}}=0\right.$, in equation (38)) when isotropic elastic behaviour is simulated in the model, which here is the case. For neutral loading, the deformation is assumed to be purely elastic. This leads to a serious drift from the yield surface already in the first integration step. If the drift correction method is not used, this initially produced drift leads to an overestimation of the undrained shear strength (Figure 4). Stability problems of this type will appear for neutral loading (or loadings close to 


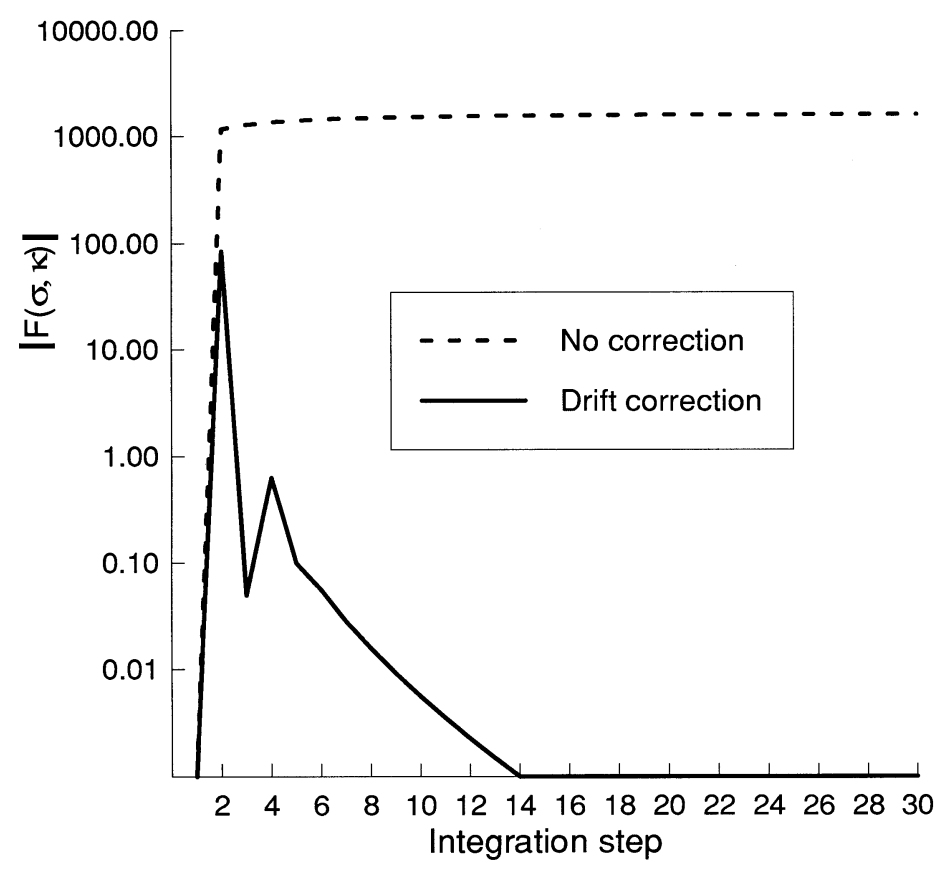

Figure 3. Yield function values under the integrations with 30 integration steps in Figure 2

neutral loading) in explicit integration algorithms because of the convex form of the yield surface. For this kind of numerical instability, very small integration increments are thus needed to avoid the problem if no drift correction method is used. Because most of the instability appears just for integration steps close to neutral loading (the first in this case, see Figure 5), smaller integration increments cannot be considered as an economical way of treating this kind of problem.

\subsection{Example 3}

In Examples 1 and 2 conventional triaxial tests have been simulated corresponding to radial loading paths in the stress space. For these two cases of simple loading some serious drift was detected, which caused e.g. an overestimation of the shear strength. However, the correct qualitative response of the simulated tests was preserved. In this example a test involving non-radial loading of a normally consolidated soil sample is simulated leading to a drift which gives rise to a qualitatively incorrect solution. The test is performed with a cyclic plastic loading close to neutral loading under drained conditions with strain control in two principal directions and stress control in the third principal direction. The integration is executed with a linear division of the control parameters between the states $\mathbf{x}^{n}$ to $\mathbf{x}^{n+1}$ and $\mathbf{x}^{n+1}$ to $\mathbf{x}^{n+2}$ according to the recursive algorithm

$$
\begin{aligned}
& \varepsilon_{1}^{n+1}=\varepsilon_{1}^{n}+0.02, \quad \varepsilon_{2}^{n+1}=\varepsilon_{2}^{n}-0.01, \quad \sigma_{3}^{n+1}=\sigma_{3}^{n} \\
& \varepsilon_{1}^{n+2}=\varepsilon_{1}^{n+1}, \quad \varepsilon_{2}^{n+2}=\varepsilon_{2}^{n+1}+0.02, \quad \sigma_{3}^{n+2}=\sigma_{3}^{n+1}
\end{aligned}
$$



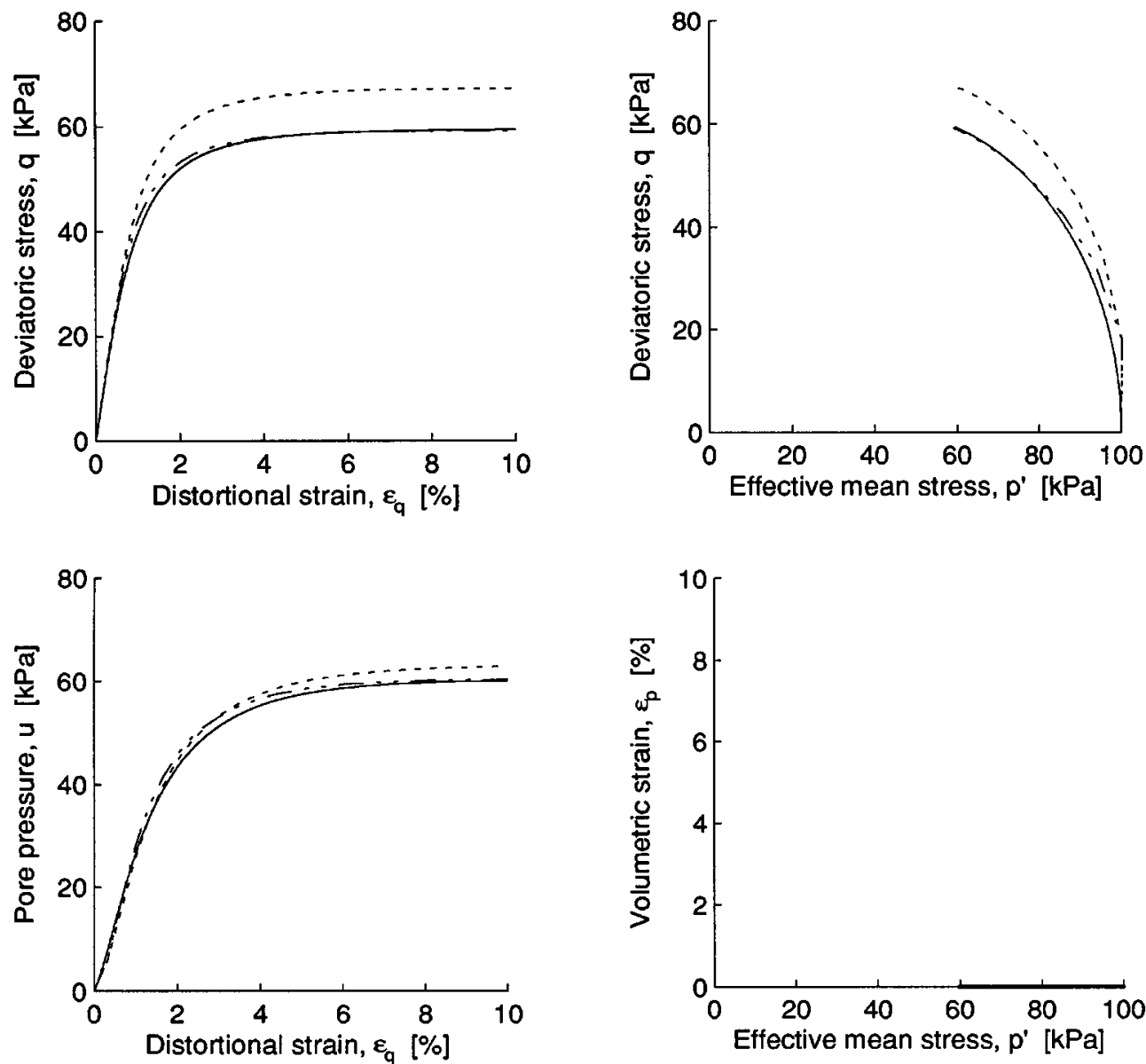

Forward Euler with 100000 integration increments

....... Forward Euler with 30 integration increments

-...- Forward Euler with 30 integration increments + drift correction method

Figure 4. A simulation of a conventional undrained triaxial compression test on a normally consolidated clay with the modified Cam Clay model

which is repeated until $n=34$, with the starting values $\varepsilon_{1}^{0}=\varepsilon_{2}^{0}=0$ and $\sigma_{3}^{0}=100$. Some results from this simulated test are shown in Figures 6 and 7. It is seen from Figure 7 that the drift, represented by the quantity $|F(\boldsymbol{\sigma}, \boldsymbol{\kappa})|$, is continuously increasing when the drift correction method is not used while it is held under control when the drift is corrected. Even here, the computation with drift correction almost corresponds to the 'exact' solution, as the responses nearly coincide in Figure 6. This example could also serve as a warning of how easily loading in different directions can destroy a calculation if the stress point is allowed to drift from the yield surface. 


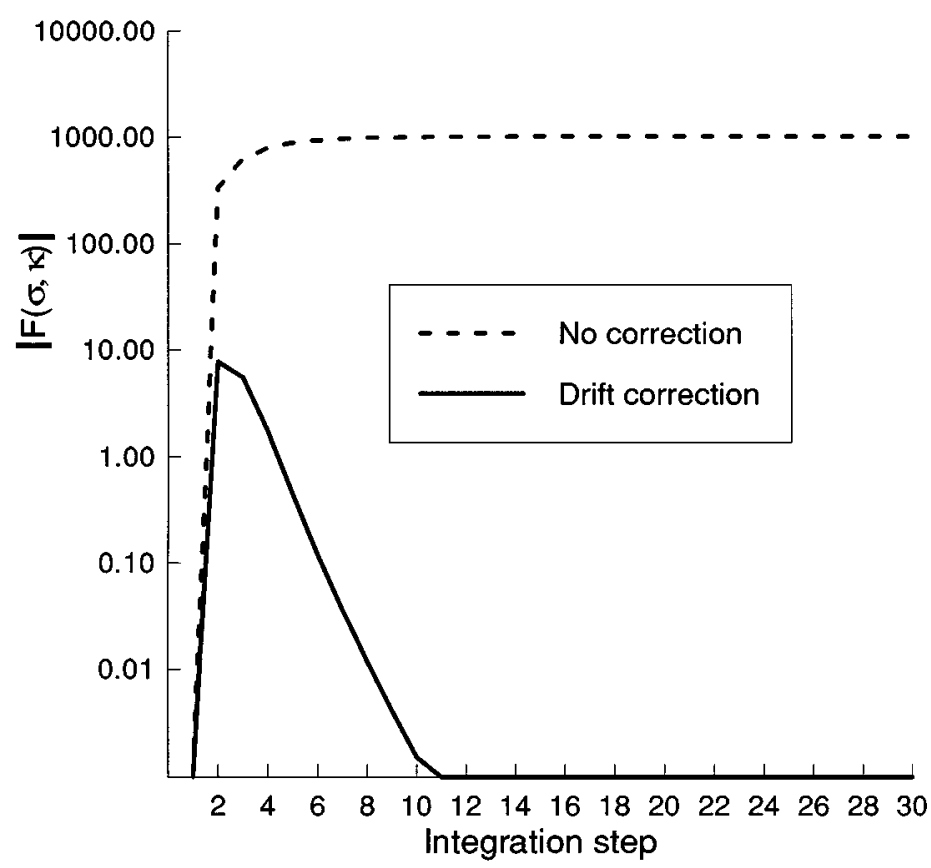

Figure 5. Yield function values under the integrations with 30 integration steps in Figure 4

\section{CONCLUDING REMARKS}

A drift correction method for explicit integration algorithms, formulated for constitutive tangential relations under mixed control, is presented. It is shown by examples that the yield surface drift severely could damage the numerical predictions while the drift correction method proposed is able to correct for the drift leading to accurate results for a small additional computational cost.

It should be emphasized that the use of small integration increments indeed is the main route of assuring good accuracy when applying explicit integration algorithms in plasticity. However, in the case of limited computer capacity or available computer time, when thus a very high number of integration steps cannot be applied, a drift correction method considerably improves the results.

In this paper, the tangential constitutive relations, on which the drift correction method is based, have been utilized in a Constitutive Driver for calibration of model parameters at the constitutive level. In such a Driver, small integration increments can be afforded and an explicit integration algorithm, e.g. forward Euler, becomes a competitive alternative to implicit algorithms, foremost because of its simplicity. However, even in a Constitutive Driver a drift correction method might be of importance. This is, for example, the case when an optimization program for identification of constitutive parameters is included in the Driver, see e.g. Reference 5. Here substantial computer effort might be needed and then the integration increments cannot be allowed to be 'too small'.

It is quite straightforward to programme the mathematics described in this paper and to adapt the formulations to a particular constitutive model. If the drift correction method is implemented as a single correction procedure (no iterations) and the values of $\partial F / \partial \boldsymbol{\sigma}, \partial G / \partial \boldsymbol{\sigma}, \partial F / \partial \mathbf{\kappa}, \mathbf{h}(\mathbf{m})$ as well as of the elastic parameters, calculated in the integration algorithm, are used to establish both the 

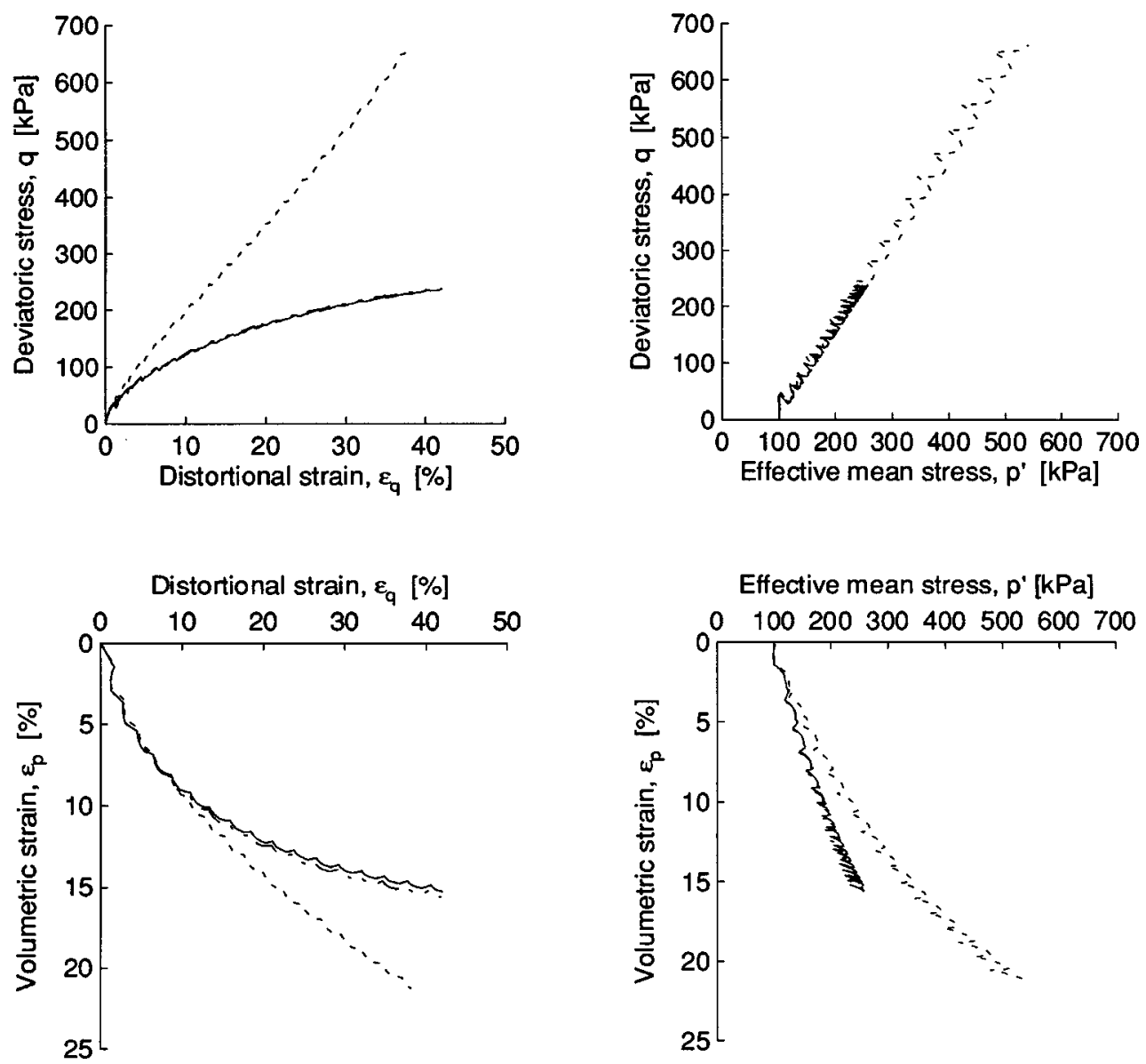

— Forward Euler with 34000 integration increments

.... Forward Euler with 170 integration increments

-...- Forward Euler with 170 integration increments + drift correction method

Figure 6. An example of plastic loading in different directions under drained conditions with the modified Cam Clay model

tangential matrix and the corrections, the additional computer time caused by the drift correction method will be very limited. For such an implementation the drift correction method is also very easy to incorporate in existing codes since most of the parameters needed already must have been determined to establish the mixed tangential relationship. In particular, $K$ in equation (21) or $K_{\mathrm{u}}$ in equation (39) is calculated as a part of the scheme and states the uniqueness of the response. In fact, the simplest form of the drift correction method described above is so inexpensive that it is difficult to find any argument not to use at least that simple form in numerical predictions. 


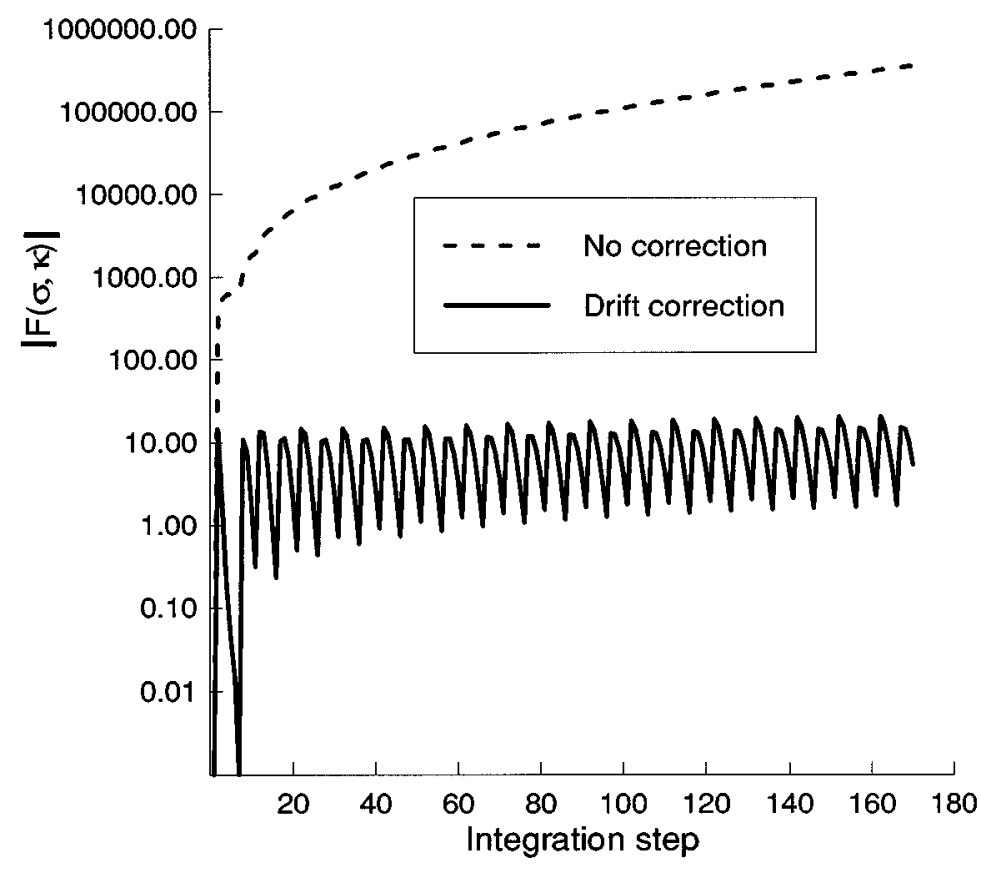

Figure 7. Yield function values under the integrations with 170 integration steps in Figure 6

\section{ACKNOWLEDGEMENTS}

Financial support from FRN (Swedish Council for Planning and Coordination of Research), for Computer Equipment, and TFR (Swedish Research Council for Engineering Science) is gratefully acknowledged. The first author also would like to thank for financial support from the Lars Erik Lundberg Foundation, Sweden.

\section{REFERENCES}

1. K. Runesson, S. Sture and K. Willam, 'Integration in computational plasticity', Comput. Struct., 30, 119-130 (1988).

2. D. M. Potts and A. Gens, 'A critical assessment of methods of correcting for drift from the yield surface in elasto-plastic finite element analysis', Int. j. numer. analyt. methods geomech., 9, 149-159 (1985).

3. M. Klisinski, Z. Mroz and K. Runesson, 'Structure of constitutive equations in plasticity for different choice of state and control variables', Int. J. Plasticity, 8, 221-243 (1992).

4. K. Runesson, K. Axelsson and M. Klisinski, 'Characteristics of constitutive relations in soil plasticity for undrained behaviour', Int. J. Solids Struct., 29, 363-380 (1992).

5. M. Klisinski, 'Optimization program for identification of constitutive parameters', Technical Report, Civil Environmental and Architectural Engineering Department, University of Colorado, BO, 1988.

6. J. S. DeNatale, 'On the calibration of constitutive models by multivariate optimization', Ph.D. Thesis, Department of Civil Engineering, University of California, Davis, 1983.

7. K. H. Roscoe and J. B. Burland, 'On the generalised stress-strain behaviour of "wet” clay', in Engineering Plasticity, Cambridge University Press, 1968, pp. 535-609.

8. D. Muir Wood, Soil Behaviour and Critical State Soil Mechanics, Cambridge University Press, Cambridge, 1990. 